TRANSACTIONS OF THE

AMERICAN MATHEMATICAL SOCIETY

Volume 362, Number 5, May 2010, Pages 2249-2277

S 0002-9947(09)05079-X

Article electronically published on December 9, 2009

\title{
NONCOMMUTATIVE POISSON STRUCTURES ON ORBIFOLDS
}

\author{
GILLES HALBOUT AND XIANG TANG
}

\begin{abstract}
In this paper, we compute the Gerstenhaber bracket on the Hochschild cohomology of $C^{\infty}(M) \rtimes G$ for a finite group $G$ acting on a compact manifold $M$. Using this computation, we obtain geometric descriptions for all noncommutative Poisson structures on $C^{\infty}(M) \rtimes G$ when $M$ is a symplectic manifold. We also discuss examples of deformation quantizations of these noncommutative Poisson structures.
\end{abstract}

\section{INTRODUCTION}

It is well known [8] that the deformation theory of an associative algebra $A$ is closely related to the Hochschild cohomology $H H^{\bullet}(A ; A)$ of $A$. In particular, the infinitesimal deformation of $A$ is governed by $H^{2}(A ; A)$. Furthermore, if we want the infinitesimal deformation to be integrable, it is necessary (but not sufficient) to require that the 2 -cocycle $\Pi \in C^{2}(A ; A)$ associated to the infinitesimal deformation satisfies the equation $[\Pi, \Pi]_{G}=0$ in $H H^{3}(A ; A)$, where $[,]_{G}$ is the Gerstenhaber bracket on $H H^{\bullet}(A ; A)$.

Suppose that $A$ is an algebra of smooth functions on a smooth manifold $M$. Then, according to the Hochschild-Kostant-Rosenberg theorem, the second Hochschild cohomology classes in $H H^{2}(A ; A)$ satisfying the above integrability conditions are in one-to-one correspondence with Poisson structures on $M$. Inspired by this relationship between Poisson geometry and deformation theory, Jonathan Block and Ezra Getzler [2] and Ping Xu [18] independently introduced a notion of a noncommutative Poisson structure on an associative algebra in the early 1990s.

Definition 1.1. A noncommutative Poisson structure on an associative algebra $A$ is an element $\Pi$ in the second Hochschild cohomology group $H^{2}(A, A)$ of $A$, whose Gerstenhaber bracket with itself vanishes, i.e. $[\Pi, \Pi]_{G}=0$.

In this paper, we want to study noncommutative Poisson structures on orbifolds coming from global quotients. Let $M$ be a compact smooth manifold, and let $G$ be a finite group acting on $M$. (For simplicity, we will always assume in this paper that the $G$-action on $M$ is effective.) Our orbifold is the quotient space $X=M / G$. Because $X$ is usually a topological space with quotient singularities, the algebra $C^{\infty}(M)^{G}$ of $G$-invariant smooth functions on $M$ is not regular. Taking a lesson from noncommutative geometry 4], we consider the crossed product algebra $C^{\infty}(M) \rtimes$

Received by the editors May 25, 2007.

2010 Mathematics Subject Classification. Primary 16E40; Secondary 58B34.

Key words and phrases. Noncommutative Poisson structure, Hochschild cohomology, Gerstenhaber bracket, deformation.

The second author was supported in part by NSF Grant 0604552.

(C)2009 American Mathematical Society 
$G$ as a natural replacement. The crossed product algebra $C^{\infty}(M) \rtimes G$, though it is noncommutative, has very nice algebraic properties. Our main aim in this paper is to find geometric descriptions for all noncommutative Poisson structures on $C^{\infty}(M) \rtimes G$ when $M$ is a symplectic manifold with a symplectic action.

As a first step toward understanding noncommutative Poisson structures on $C^{\infty}(M) \rtimes G$, the second author and his coauthors 13 computed the Hochschild cohomology of $C^{\infty}(M) \rtimes G$ as a vector space:

$$
H H^{\bullet}\left(C^{\infty}(M) \rtimes G ; C^{\infty}(M) \rtimes G\right)=\Gamma^{\infty}\left(\bigoplus_{g \in G} \wedge^{\bullet-l(g)} T M^{g} \otimes \wedge^{l(g)} N^{g}\right)^{G} .
$$

We explain the notation in the above equation. First, $M^{g}$ is the fixed point manifold of $g$, and $N^{g}$ is the normal bundle of the embedding of $M^{g}$ in $M$, where $l(g)$ is the dimension of $N^{g}$. The group $G$ acts on the disjoint union $\bigsqcup M^{g}$ of $M^{g}$ for all $g \in G$ by the conjugate action. We remark that $M^{g}$ may have different components with different dimensions, and we take the disjoint union of all the components, and accordingly $l(g)$ is taken as a local constant function on $M^{g}$. (Following [13], in this paper we view $C^{\infty}(M) \rtimes G$ as a bornological algebra with the bornology defined by the Fréchet topology. We take $H H^{\bullet}$ to be the continuous Hochschild cohomology of a bornological algebra. Accordingly, all computations and constructions in this paper are local with respect to the orbifold $M / G$. We often work with a vector space (or a $G$-invariant open subset) with a linear $G$-action, which we refer to as "local" computation.) We call the stratified space $\bigsqcup M^{g} / G$ the inertia orbifold $\tilde{X}$ associated to $X=M / G$. In other words, we can interpret Equation (11) as saying that the Hochschild cohomology of $C^{\infty}(M) \rtimes G$ is equal to the space of "vector fields" on $\tilde{X}$.

The main difficulty in finding noncommutative Poisson structures on $C^{\infty}(M) \rtimes G$ is to compute the Gerstenhaber bracket on $H H^{\bullet}\left(C^{\infty}(M) \rtimes G ; C^{\infty}(M) \rtimes G\right)$. To compute the Gerstenhaber bracket, we need to have quasi-isomorphisms between the Hochschild cochain complex

$$
C^{\bullet}\left(C^{\infty}(M) \rtimes G ; C^{\infty}(M) \rtimes G\right)
$$

and

$$
\Gamma^{\infty}\left(\bigoplus_{g \in G} \wedge^{\bullet-l(g)} T M^{g} \otimes \wedge^{l(g)} N^{g}\right)^{G} .
$$

In [13], we defined a quasi-isomorphism $L$ in the following direction:

$$
L: C^{\bullet}\left(C^{\infty}(M) \rtimes G ; C^{\infty}(M) \rtimes G\right) \longrightarrow \Gamma^{\infty}\left(\bigoplus_{g \in G} \wedge^{\bullet-l(g)} T M^{g} \otimes \wedge^{l(g)} N^{g}\right)^{G} .
$$

The first step of this paper is to define a quasi-isomorphism $T$ in the other direction. This turns out to be much harder to construct than the map $L$ in [13]. We need to construct some nonlocal operators on $C^{\infty}(M)$, which we call twisted cocycles. These cocycles are closely related to the Lusztig-Demazure operator (cf. [14). Using the maps $T$ and $L$, we are able to compute the Gerstenhaber brackets on $H H^{\bullet}\left(C^{\infty}(M) \rtimes G ; C^{\infty}(M) \rtimes G\right)$. Our results show that the Gerstenhaber bracket on orbifolds is a generalization of the classical Schouten-Nijenhuis bracket on manifolds. We call this bracket the twisted Schouten-Nijenhuis bracket on $\Gamma^{\infty}\left(\bigoplus_{g} \wedge^{\bullet-l(g)} T M^{g} \otimes \wedge^{l(g)} N^{g}\right)^{G}$. Using the twisted Schouten-Nijenhuis bracket, we are able to solve the equation $[\Pi, \Pi]_{G}=0$ on $H H^{2}\left(C^{\infty}(M) \rtimes G ; C^{\infty}(M) \rtimes G\right)$ 
geometrically. This leads to a full description of noncommutative Poisson structures on $C^{\infty}(M) \rtimes G$ when $M$ is a symplectic manifold.

If we consider a complex symplectic vector space $V$ with a symplectic $G$-action, the cocycles used in the definition of symplectic reflection algebras [7 correspond to a special class of noncommutative Poisson structures on $\operatorname{Poly}(V) \rtimes G$, where $\operatorname{Poly}(V)$ is the algebra of polynomials on $V$. Using the results from [7] we prove in this paper that all these cocycles can be extended to a formal deformation of the algebra $\operatorname{Poly}(V) \rtimes G$. As a generalization, we expect that all the noncommutative Poisson structures discovered in this paper can be extended to formal deformations, which will generalize the symplectic reflection algebras. This question is closely related to the following formality conjecture on orbifolds, which we will discuss in future publications.

Conjecture 1.2. The Hochschild complex of the algebra $C^{\infty}(M) \rtimes G$ is a formal differential graded Lie algebra.

In the last part of this paper, we provide concrete new examples of noncommutative Poisson structures on $\operatorname{Poly}\left(\mathbb{R}^{4}\right) \rtimes\left(\mathbb{Z}_{n} \times \mathbb{Z}_{m}\right)$ with $\mathbb{Z}_{n}=\mathbb{Z} / \mathbb{Z}_{n}$ and $\mathbb{Z}_{m}=\mathbb{Z} / m \mathbb{Z}$. These Poisson structures are not symplectic at all, and instead should be viewed as noncommutative quadratic Poisson structures. The connection between these "noncommutative quadratic Poisson structures" and quantum $R$-matrices will be studied in the near future. In general, there are many interesting examples of noncommutative Poisson structures on orbifolds. We are working with Jean-Michel Oudom in [11] on this material.

Besides the Gerstenhaber bracket, there is also a product on the Hochschild cohomology $H H^{\bullet}\left(C^{\infty}(M) \rtimes G ; C^{\infty}(M) \rtimes G\right)$. In [15], with Pflaum, Posthuma and Tseng, the second author studied the ring structure on the Hochschild cohomology of the deformed algebras of $C^{\infty}(M) \rtimes G$, which is closely related to the Chen-Ruan orbifold cohomology $[3]$.

This paper is organized as follows. In Section 2, we briefly recall the basic definitions in Hochschild cohomology and the computations of $H H^{\bullet}\left(C^{\infty}(M) \rtimes\right.$ $\left.G, C^{\infty}(M) \rtimes G\right)$ as a vector space in 13. In Section 3, we will focus on the construction of twisted cocycles and a quasi-isomorphism $T$,

$$
T: \Gamma^{\infty}\left(\bigoplus_{g \in G} \wedge^{\bullet-l(g)} T M^{g} \otimes \wedge^{l(g)} N^{g}\right)^{g} \longrightarrow C^{\bullet}\left(C^{\infty}(M) \rtimes G ; C^{\infty}(M) \rtimes G\right) .
$$

In Section 4, we will study the Gerstenhaber bracket on the Hochschild cohomology $H H^{\bullet}\left(C^{\infty}(M) \rtimes G ; C^{\infty}(M) \rtimes G\right)$. In Section 5, we will give a full description of noncommutative Poisson structures on $C^{\infty}(M) \rtimes G$ when $M$ is a symplectic manifold. Furthermore we will study formal deformations of a special type of noncommutative Poisson structures and compute their second Poisson cohomology group. We end this section by showing two new families of noncommutative quadratic Poisson structures on $\mathbb{C}^{2} / \mathbb{Z}_{n} \times \mathbb{Z}_{m}$.

Remark 1.3. Unless otherwise specified, we work with the field $\mathbb{R}$, real vector spaces, and real manifolds. However, many results in this paper have analogs with the field $\mathbb{C}$, complex vector spaces and affine varieties.

\section{Hochschild COHOMOLOGY OF $C^{\infty}(M) \rtimes G$}

In this section, we briefly review our work in [13] on computing the Hochschild cohomology of the algebra $C^{\infty}(M) \rtimes G$ as a vector space. 
2.1. Hochschild cohomology of an algebra. We review in this subsection the definition of Hochschild cohomology of an algebra. Let $A$ be a unital algebra over $\mathbb{R}$, and let $M$ be a bimodule of $A$. The degree $n$ Hochschild cochain complex $C^{n}(A, M)$ of $A$ with coefficients in $M$ consists of $\mathbb{R}$-linear maps from $A^{\otimes n}$ to $M$, i.e. $\operatorname{Hom}\left(A^{\otimes n}, M\right)$. The coboundary map $\partial$ on Hochschild cochains $C^{\bullet}(A, M)$ is defined by

$$
\begin{gathered}
\partial: \quad C^{n}(A, M) \longrightarrow C^{n+1}(A, M) \\
\partial(\xi)\left(a_{0}, \cdots, a_{n}\right)=a_{0} \xi\left(a_{1}, \cdots, a_{n}\right)+\sum_{i=1}^{n}(-1)^{i} \xi\left(\cdots, a_{i-1} a_{i}, \cdots\right) \\
+(-1)^{n+1} \xi\left(a_{1}, \cdots, a_{n-1}\right) a_{n} .
\end{gathered}
$$

It is a straightforward check that $\partial^{2}=0$.

Definition 2.1. Define the Hochschild cohomology of $A$ with coefficient in $M$ to be the cohomology of the differential cochain complex $\left(C^{\bullet}(A, M), \partial\right)$.

Remark 2.2. In the case that $A$ is a topological (or bornological) algebra, then we need to consider a topological (bornological) algebra bimodule $M$, and the topological (bornological) tensor product of $A$, and continuous (bounded) linear maps from $A$ to $M$. All the definitions we introduce below naturally extend to the topological (bornological) version and we refer to [15, Appendix A] for details. The above definitions work for arbitrary fields.

The left and right multiplication of $A$ on $A$ makes $A$ a bimodule of $A$. Gerstenhaber [8] used the Hochschild cohomology $H^{\bullet}(A, A)$ to study deformation theory of $A$. On $C^{\bullet}(A, A)$, besides the coboundary differential, there are two interesting operations:

(1) the Gerstenhaber bracket, and

(2) the cup product.

The Gerstenhaber bracket is used in defining a noncommutative Poisson structure in Definition 1.1. We recall its definition.

Definition 2.3. We define a pre-Lie product $\circ$ on $C^{\bullet}(A ; A)$. For $\phi \in C^{k}(A ; A)$, $\psi \in C^{l}(A ; A), \phi \circ \psi \in C^{k+l-1}(A ; A)$ is defined by

$$
\begin{aligned}
& \phi \circ \psi\left(a_{1}, \ldots, a_{k+l-1}\right) \\
= & \sum_{i=1}^{k}(-1)^{(i-1)(l-1)} \phi\left(a_{1}, \ldots, a_{i-1}, \psi\left(a_{i}, \ldots, a_{i+l-1}\right), a_{i+l}, \ldots, a_{k+l-1}\right),
\end{aligned}
$$

for $a_{i} \in A, i=1, \ldots, k+l-1$. The Gerstenhaber bracket [ , ] on $C^{\bullet}(A ; A)$ is defined to be the commutator of the pre-Lie product, i.e.,

$$
[\phi, \psi]_{G}=\phi \circ \psi-(-1)^{(k-1)(l-1)} \psi \circ \phi .
$$

The Gerstenhaber bracket is compatible with the differential on $C^{\bullet}(A, A)$, and therefore defines a Lie bracket on the Hochschild cohomology $H H^{\bullet}(A, A)$.

2.2. $H H^{\bullet}(\operatorname{Poly}(V) \rtimes G, \operatorname{Poly}(V) \rtimes G)$. In this subsection, we consider the algebra $C^{\infty}(M) \rtimes G$ for a finite group action on a compact manifold $M$, and explain the computation in [13] of the Hochschild cohomology $H H^{\bullet}\left(C^{\infty}(M) \rtimes G, C^{\infty}(M) \rtimes G\right)$. In this subsection we will mainly focus on the local case, i.e., the $G$-linear action on a 
vector space $V$; in the next subsection, we explain the extension of the computations to general manifolds.

Let $\operatorname{Poly}(V)$ be the algebra of polynomial functions on a vector space $V$, and let $G$ be a finite group acting linearly on $V$. We computed [13] the Hochschild cohomology of the crossed product algebra $\operatorname{Poly}(V) \rtimes G$. The major step is a construction of a quasi-isomorphism

$$
L: C^{\bullet}(\operatorname{Poly}(V) \rtimes G, \operatorname{Poly}(V) \rtimes G) \longrightarrow \Gamma^{\infty}\left(\bigoplus_{g \in G} \wedge^{\bullet-l(g)} T V^{g} \otimes \wedge^{l(g)} N^{g}\right)^{G} .
$$

This map was constructed implicitly in the proof of Theorem 3.1 in [13. We make it explicit in the following.

The map $L$ is a composition of three cochain maps:

Step I.

$$
L_{1}: C^{\bullet}(\operatorname{Poly}(V) \rtimes G, \operatorname{Poly}(V) \rtimes G) \longrightarrow\left(C^{\bullet}(\operatorname{Poly}(V), \operatorname{Poly}(V) \rtimes G)\right)^{G},
$$

where $G$ acts on $C^{\bullet}(\operatorname{Poly}(V), \operatorname{Poly}(V) \rtimes G)$ by

$$
g \Psi\left(a_{1}, \ldots, a_{n}\right)=U_{g^{-1}} \cdot \Psi\left(g\left(a_{1}\right), \ldots, g\left(a_{n}\right)\right) \cdot U_{g} .
$$

Here $U_{g}$ denotes the element $g$ seen in $\operatorname{Poly}(V) \rtimes G$. Given a Hochschild cocycle $\Psi \in C^{k}(\operatorname{Poly}(V) \rtimes G, \operatorname{Poly}(V) \rtimes G)$, we define $L_{1}(\Psi) \in\left(C^{k}(\operatorname{Poly}(V), \operatorname{Poly}(V) \rtimes\right.$ $G))^{G}$ by

$$
L_{1}(\Psi)\left(f_{1}, \ldots, f_{k}\right)=\frac{1}{|G|} \sum_{g}(g \Psi)\left(f_{1}, \ldots, f_{k}\right), \quad \forall f_{1}, \ldots, f_{k} \in \operatorname{Poly}(V),
$$

where $|G|$ is the order of the group $G$.

Step II.

$$
L_{2}:\left(C^{\bullet}(\operatorname{Poly}(V), \operatorname{Poly}(V) \rtimes G)\right)^{G} \longrightarrow\left(\bigoplus_{g \in G} \Gamma^{\infty}\left(\wedge^{\bullet} T V\right), \kappa_{g} \wedge\right)^{G} .
$$

Let $A_{g}$ be a vector space isomorphic to $\operatorname{Poly}(V)$ equipped with the $\operatorname{Poly}(V)$ bimodule structure

$$
a \cdot \xi \cdot(b)=a \xi g(b), \text { for } a, b \in \operatorname{Poly}(V), \xi \in \operatorname{Poly}(V)_{g},
$$

where the right hand side is the product of $a, \xi$, and $g(b)$ as elements in $\operatorname{Poly}(V)$. As a $\operatorname{Poly}(V)-\operatorname{Poly}(V)$ bimodule, $\operatorname{Poly}(V) \rtimes G$ has a natural splitting into a direct sum of submodules $\bigoplus_{g \in G} A_{g}$. Correspondingly, the cochain complex $C^{\bullet}(\operatorname{Poly}(V)$, $\operatorname{Poly}(V) \rtimes G)$ has a natural splitting into $\bigoplus_{g \in G} C^{\bullet}\left(\operatorname{Poly}(V), A_{g}\right)$. We define $L_{2}$ to be the sum of the maps

$$
L_{2}^{g}: C^{\bullet}\left(\operatorname{Poly}(V), A_{g}\right) \longrightarrow\left(\Gamma^{\infty}\left(\wedge^{\bullet} T V\right), \kappa_{g}\right)
$$

over all $g \in G$.

On $V$, we introduce the vector field $X(x)=\sum_{i} x^{i} \frac{\partial}{\partial x^{i}}$, where the $x^{i}$ are coordinate functions on $V$. Define the vector field $\kappa_{g} \in \Gamma^{\infty}(T V)$ by

$$
\kappa_{g}(x)=X(g(x))-X(x) .
$$

We notice that for a permutation $\sigma$ of $k$ elements fixing $x \in V$, the product $\left(x_{\sigma(1)}^{i_{1}}-x^{i_{1}}\right) \cdots\left(x_{\sigma(k)}^{i_{k}}-x^{i_{k}}\right)$ is a function on $x_{1}, \ldots, x_{k} \in V$ by taking the product of the values of the coordinate functions. Given an element $\Psi \in C^{k}\left(\operatorname{Poly}(V), A_{g}\right)$, we 
define $L_{2}^{g}(\Psi) \in \Gamma^{\infty}\left(\wedge^{k} T V\right)$, the usual projection to anti-symmetric linear operators, by

$$
\begin{gathered}
L_{2}^{g}(\Psi)(x)=\sum_{i_{1}, \ldots, i_{k}} \frac{1}{k !} \sum_{\sigma \in S_{k}}(-1)^{\sigma} \Psi\left(\left(x_{\sigma(1)}\right)^{i_{1}}-x^{i_{1}}\right) \cdots \\
\left.\left(x_{\sigma(k)}^{i_{k}}-x^{i_{k}}\right)\right)(x) \frac{\partial}{\partial x^{i_{1}}} \wedge \cdots \wedge \frac{\partial}{\partial x^{i_{k}}},
\end{gathered}
$$

where $S_{k}$ is the permutation group of $k$ elements.

The $G$-action on $\left(\bigoplus_{g \in G} \Gamma^{\infty}\left(\wedge^{\bullet} T V\right), \kappa_{g} \wedge\right)$ is defined by

$$
\left.h\left(\sum_{g} \phi_{g}\right)\right|_{h^{-1} g h}=h_{*}\left(\phi_{g}\right), \quad \text { for } \sum_{g} \phi_{g} \in \bigoplus_{g} \Gamma^{\infty}\left(\wedge^{\bullet} T V\right) \text {, and } h \in G .
$$

It is straightforward to check that $L_{2}^{g}$ is $G$-equivariant and therefore defines a map

$$
L_{2}:\left(C^{\bullet}(\operatorname{Poly}(V), \operatorname{Poly}(V) \rtimes G)\right)^{G} \longrightarrow\left(\bigoplus_{g \in G} \Gamma^{\infty}\left(\wedge^{\bullet} T V\right), \kappa_{g} \wedge\right)^{G} .
$$

Step III.

$$
L_{3}:\left(\bigoplus_{g \in G}\left(\Gamma^{\infty}\left(\wedge^{\bullet} T V\right), \kappa_{g} \wedge\right)\right)^{G} \longrightarrow\left(\bigoplus_{g \in G}\left(\Gamma^{\infty}\left(\wedge^{\bullet-l(g)} T V^{g} \otimes \wedge^{l(g)} N^{g}\right), 0\right)\right)^{G} .
$$

Let $C_{g}$ be the cyclic group generated by $g$, which has a natural action on $V$. As $C_{g}$ is abelian, $V$ is decomposed into a direct sum of $C_{g}$ irreducible representations. Let $V^{g}$ be the subspace of all trivial $C_{g^{-}}$-representations in $V$, and $N^{g}$ be the sum of all nontrivial irreducible $C_{g^{-}}$-representations in $V$. Therefore, $V$ can be written as $V^{g} \oplus N^{g}$.

We define $L_{3}$ to be the sum of $L_{3}^{g}$, which is defined to be

$$
L_{3}^{g}(X)=p r^{g}\left(\left.X\right|_{V^{g}}\right),
$$

where $\left.X\right|_{V^{g}}$ is the restriction of $X \in \wedge^{\bullet} T V$ to $\left.\wedge^{\bullet} T V\right|_{V^{g}}$, and $p r^{g}$ projects $\left.\wedge^{\bullet} T V\right|_{V^{g}}$ to $\wedge^{\bullet-l(g)} T V^{g} \otimes \wedge^{l(g)} N^{g}$.

The space $\bigoplus_{g \in G}\left(\Gamma^{\infty}\left(\wedge^{\bullet-l(g)} T V^{g} \otimes \wedge^{l(g)} N^{g}, 0\right)\right.$ is closed under $G$-action on $\left(\bigoplus_{g \in G} \Gamma^{\infty}\left(\wedge^{\bullet} T V\right), \kappa_{g} \wedge\right)$ and therefore inherits a $G$-action. Similarly to the computations taken in Step II, we can easily check that $L_{3}^{g}$ is $G$-equivariant and that therefore $L_{3}$ defines a map on the $G$-invariant components.

We proved in Section 3 of [13] that $L=L_{3} \circ L_{2} \circ L_{1}$ is a quasi-isomorphism of cochain complexes

$$
L: C^{\bullet}(\operatorname{Poly}(V) \rtimes G, \operatorname{Poly}(V) \rtimes G) \longrightarrow \Gamma^{\infty}\left(\bigoplus_{g \in G} \wedge^{\bullet-l(g)} T V^{g} \otimes \wedge^{l(g)} N^{g}\right)^{G} .
$$

2.3. The general cases. In the above Steps I-III, we have explained how to compute the Hochschild cohomology of $\operatorname{Poly}(V) \rtimes G$ using the quasi-isomorphism $L$. We explain briefly how to generalize this construction to $C^{\infty}(M) \rtimes G$ by defining $L$ to be

$$
L: C^{\bullet}\left(C^{\infty}(M) \rtimes G, C^{\infty}(M) \rtimes G\right) \longrightarrow \Gamma^{\infty}\left(\bigoplus_{g \in G} \wedge^{\bullet-l(g)} T M^{g} \otimes \wedge^{l(g)} N^{g}\right)^{G} .
$$


Firstly, we observe that the map $L_{1}$ is a quasi-isomorphism from $C^{\bullet}(A \rtimes G, A \rtimes G)$ to $\left(C^{\bullet}(A, A \rtimes G)\right)^{G}$ that is true for any algebra $A$ with a finite group action. Therefore, the map $L_{1}$ extends to the general case $C^{\infty}(M) \rtimes G$ naturally:

$$
L_{1}: C^{\bullet}\left(C^{\infty}(M) \rtimes G, C^{\infty}(M) \rtimes G\right) \longrightarrow\left(C^{\bullet}\left(C^{\infty}(M), C^{\infty}(M) \rtimes G\right)\right)^{G} .
$$

Secondly, $L_{3}$ is generalized to the manifold case, as the map

$$
L_{3}:\left(\bigoplus_{g \in G}\left(\Gamma^{\infty}\left(\wedge^{\bullet} T M\right), \kappa \wedge\right)\right)^{G} \longrightarrow\left(\bigoplus_{g \in G}\left(\Gamma^{\infty}\left(\wedge^{\bullet-l(g)} \otimes \wedge^{l(g)} N^{g}\right), 0\right)\right)^{G}
$$

obtained by composing the projection map $\left.\wedge^{\bullet} T M\right|_{M^{g}} \rightarrow \wedge^{\bullet-l(g)} T M^{g} \otimes \wedge^{l(g)} N^{g}$ with the restriction map from $\wedge^{\bullet} T M$ to $\left.\wedge^{\bullet} T M\right|_{V}$.

Thirdly, to generalize $L_{2}$, we use Connes' map [4, Lemma 44] from the Koszul resolution of $C^{\infty}(M)$ to its bar resolution for a manifold with an affine structure. We can then define $L_{2}^{g}$ from the Hochschild cochain complex $C^{\bullet}\left(C^{\infty}(M), C^{\infty}(M)_{g}\right)$ to $\wedge^{\bullet} T M$ in a similar way. There is a small issue here that the Connes construction 4. Lemma 44] only works for affine manifolds. Therefore, for a general manifold $M$, we need to realize the Hochschild cochain complex of $C^{\infty}(M) \rtimes G$ as a (pre)sheaf over the orbifold $M / G$, and use Čech techniques to compute the sheaf cohomology of this (pre)sheaf. In this framework, $L$ will be a quasi-isomorphism of (pre)sheaves which is locally defined as the $L_{g}$ was in Section 2.2, Step II. We refer to [15, Section 3] for details.

At the end of this subsection, we explain the following observation which makes our study easier. Recall Equation (11) concerning the Hochschild cohomology,

$$
H H^{\bullet}\left(C^{\infty}(M) \rtimes G ; C^{\infty}(M) \rtimes G\right)=\Gamma^{\infty}\left(\bigoplus_{g \in G} \wedge^{\bullet-l(g)} T M^{g} \otimes \wedge^{l(g)} N^{g}\right)^{G}
$$

As $g$ is in the $g$-centralizer subgroup of $G$, the $g$-fixed point component's contribution to $H H^{\bullet}\left(C^{\infty}(M) \rtimes G, C^{\infty}(M) \rtimes G\right)$ has to be from $g$-invariant sections of $\Gamma^{\infty}\left(\wedge^{\bullet-l(g)} T V^{g} \otimes \wedge^{l(g)} N^{g}\right)$. As $g$ acts on $T V^{g}$ trivially, a $g$-invariant section of $\wedge^{\bullet-l(g)} T V^{g} \otimes \wedge^{l(g)} N^{g}$ must have $g$-invariant component in $\wedge^{l(g)} N^{g}$. Note that $\wedge^{l(g)} N^{g}$ is a line bundle over $V^{g}$. To have a nonzero $g$-invariant section, we need that the $g$-action on $\wedge^{l(g)} N^{g}$ must be trivial. This implies that $\operatorname{det}\left(\left.g\right|_{N^{g}}\right)=1$. Furthermore, we notice that $\left.g\right|_{N^{g}}$ 's action on $N^{g}$ is of finite order and therefore can be diagonalized. If $N^{g}$ is of odd dimension, then by the fact that $\operatorname{det}\left(\left.g\right|_{N^{g}}\right)=1$, we conclude that $\left.g\right|_{N^{g}}$ has at least one eigenvalue equal to 1 . This contradicts the assumption of $N^{g}$. Therefore, we conclude that if $\operatorname{dim}\left(N^{g}\right)$ is odd, there is no nonzero contribution to $H H^{\bullet}\left(C^{\infty}(M) \rtimes G, C^{\infty}(M) \rtimes G\right)$ from this $g$-fixed point component. Hence, the Hochschild cohomology of $C^{\infty}(M) \rtimes G$ has no contribution from $g$-fixed point submanifolds with odd $l(g)$. Therefore, we conclude

(2)

$$
H H^{\bullet}\left(C^{\infty}(M) \rtimes G ; C^{\infty}(M) \rtimes G\right)=\Gamma^{\infty}\left(\underset{\substack{g \in G, l(g) \text { is even }}}{\bigoplus} \wedge^{\bullet-l(g)} T M^{g} \otimes \wedge^{l(g)} N^{g}\right)^{G} .
$$




\section{HoCHSCHILD COHOMOLOGY AND QUASI-ISOMORPHISMS}

In this section, we construct a quasi-isomorphism

$$
T: \Gamma^{\infty}\left(\bigoplus_{g \in G} \wedge^{\bullet-l(g)} T M^{g} \otimes \wedge^{l(g)} N^{g}\right)^{G} \longrightarrow C^{\bullet}\left(C^{\infty}(M) \rtimes G ; C^{\infty}(M) \rtimes G\right),
$$

which is a quasi-inverse to the map $L$ reviewed in Sections 2.2-2.3. First, we construct a twisted cocycle $\Omega_{g}$ for each element $g$ associated to the determinant line bundle $\wedge^{l(g)} N^{g}$; second, we use the twisted cocycles to construct the map $T$. We mainly focus on the local case $\operatorname{Poly}(V) \rtimes G$ and explain at the end how to generalize the construction to general manifolds.

3.1. Twisted cocycle. To construct the map $T$, we need to understand cocycles in $C^{\bullet}\left(\operatorname{Poly}(V), A_{g}\right)$, which we call $g$-twisted cocycles. (Recall that $A_{g}$ is a bimodule of $\operatorname{Poly}(V)$ isomorphic to $\operatorname{Poly}(V)$ as a vector space but with the $g$-twisted multiplication from the right (Section 2.2, Step II).) If we trace through the computation Steps I-III in Section 2.2, we see that $H^{\bullet}\left(\operatorname{Poly}(V), A_{g}\right)$ is isomorphic to $\Gamma^{\infty}\left(\wedge^{\bullet-l(g)} T V^{g} \otimes \otimes \wedge^{l(g)} N^{g}\right)$. To construct the quasi-inverse of $L$, we need to associate to each element in $\Gamma^{\infty}\left(\wedge^{\bullet-l(g)} T V^{g} \otimes \wedge^{l(g)} N^{g}\right)$ a cocycle in $C^{\bullet}\left(\operatorname{Poly}(V), A_{g}\right)$. In particular, we need to associate to an element in $\wedge^{l(g)} N^{g}$ a degree $l(g)$ cocycle in $C^{\bullet}\left(\operatorname{Poly}(V), A_{g}\right)$. It is well known that an element in $\Gamma^{\infty}\left(\wedge^{\bullet-l(g)} T V^{g} \otimes \wedge^{l(g)} N^{g}\right)$ can be viewed as a multi-differential operator on $V$. A natural guess is that this multi-differential operator might be a cocycle in $C^{\bullet}\left(\operatorname{Poly}(V), A_{g}\right)$. However, one can easily check that such a multi-differential operator is not a cocycle in $C^{\bullet}\left(\operatorname{Poly}(V), A_{g}\right)$ except in the case $g=1$. (For instance, because the right module structure on $A_{g}$ is twisted by the $g$-action, a degree 1 cocycle $\xi$ in $C^{\bullet}\left(\operatorname{Poly}(V), A_{g}\right)$ must satisfy $\xi(a b)=a \xi(b)+\xi(a) g(b)$. One can quickly check that when $g \neq 1$, no nonzero vector fields on $V$ can satisfy such an equation.) Therefore, we have to modify the above natural guess so that the outcome is a cocycle in $C^{\bullet}\left(\operatorname{Poly}(V), A_{g}\right)$. This leads us to the following construction.

Recall that $V=V^{g} \oplus N^{g}$. We choose coordinates $x^{1}, \ldots, x^{n-l(g)}, y^{1}, \ldots, y^{l(g)}$ on $V(n=\operatorname{dim}(V))$, such that $x^{1}, \ldots, x^{n-l(g)}$ are coordinates on $V^{g}$ and $y^{1}, \ldots, y^{l(g)}$ are coordinates on $N^{g}$. We assume that the $g$-action on $y^{1}, \ldots, y^{l(g)}$ is diagonalized 1 .

For any $\sigma \in S_{l(g)}$, the permutation group of $l(g)$ elements, we introduce the following vectors in $N^{g}$. Let $\left(\tilde{y}^{1}, \ldots, \tilde{y}^{l(g)}\right)=g\left(y^{1}, \ldots y^{l(g)}\right)$. Define

$$
\begin{array}{cc}
z_{\sigma}^{0}=\left(y^{1}, \ldots, y^{l(g)}\right), & z_{\sigma}^{1}=\left(y^{1}, \ldots, \tilde{y}^{\sigma(1)}, \ldots\right), \\
z_{\sigma}^{2}=\left(y^{1}, \ldots, \tilde{y}^{\sigma(1)}, \ldots, \tilde{y}^{\sigma(2)}, \ldots\right), & \\
z_{\sigma}^{l(g)-1}=\left(\tilde{y}^{1}, \ldots, y^{\sigma(l(g))}, \ldots\right), & z_{\sigma}^{l(g)}=\left(\tilde{y}^{1}, \ldots, \tilde{y}^{l(g)}\right) .
\end{array}
$$

Let $\Omega_{g}$ be an $l(g)$-cochain in $C^{\bullet}\left(A, A_{g}\right)$ defined as follows:

$$
\begin{aligned}
& \Omega_{g}\left(f_{1}, \ldots, f_{l(g)}\right)(x, y):=\frac{1}{l(g) !} \sum_{\sigma \in S_{l(g)}}(-1)^{\sigma} \\
& . \frac{\left(f_{1}\left(x, z_{\sigma}^{0}\right)-f_{1}\left(x, z_{\sigma}^{1}\right)\right)\left(f_{2}\left(x, z_{\sigma}^{1}\right)-f_{2}\left(x, z_{\sigma}^{2}\right)\right) \cdots\left(f_{l(g)}\left(x, z_{\sigma}^{l(g)-1}\right)-f_{l(g)}\left(x, z_{\sigma}^{l(g)}\right)\right)}{\left(y^{1}-\tilde{y}^{1}\right) \cdots\left(y^{l(g)}-\tilde{y}^{l(g)}\right)} .
\end{aligned}
$$

\footnotetext{
${ }^{1}$ We can pass to the complex coordinates to achieve such a choice.
} 
We remark that when $\sigma$ is the identity permutation, we have

$$
\begin{gathered}
z_{i d}^{0}=\left(y^{1}, \ldots, y^{l(g)}\right), \quad z_{i d}^{1}=\left(\tilde{y}^{1}, \ldots, y^{l(g)}\right), \\
\ldots \ldots \\
z_{i d}^{l(g)-1}=\left(\tilde{y}^{1}, \ldots, \tilde{y}^{l(g)-1}, y^{l(g)}\right), \quad z_{i d}^{l(g)}=\left(\tilde{y}^{1}, \ldots, \tilde{y}^{l(g)}\right) .
\end{gathered}
$$

The corresponding contribution in the summation of expression (3) is

$\frac{\left(f_{1}\left(x, z_{i d}^{0}\right)-f_{1}\left(x, z_{i d}^{1}\right)\right)\left(f_{2}\left(x, z_{i d}^{1}\right)-f_{2}\left(x, z_{i d}^{2}\right)\right) \cdots\left(f_{l(g)}\left(x, z_{i d}^{l(g)-1}\right)-f_{l(g)}\left(x, z_{i d}^{l(g)}\right)\right)}{\left(y^{1}-\tilde{y}^{1}\right) \cdots\left(y^{l(g)}-\tilde{y}^{l(g)}\right)}$

which converges to

$$
\frac{\partial}{\partial y^{1}} f_{1}(x, 0) \cdots \frac{\partial}{\partial y^{l(g)}} f_{l(g)}(x, 0)
$$

as $y^{1}, \ldots, y^{l(g)}$ go to 0 .

Therefore the identity component in Equation (3) can be viewed as a $g$ analog of the multi-differential operator

$$
\frac{\partial}{\partial y^{1}} \otimes \cdots \otimes \frac{\partial}{\partial y^{l(g)}}
$$

Summing over all permutations, $\Omega_{g}$ can be viewed as a $g$-analog of the multidifferential operator

$$
\Lambda_{g}=\frac{\partial}{\partial y^{1}} \wedge \cdots \wedge \frac{\partial}{\partial y^{l(g)}}
$$

It is straightforward to check that $\Omega_{g}$ is a cocycle in $C^{l(g)}\left(A, A_{g}\right)$.

The following is a simple property of $\Omega_{g}$.

Lemma 3.1. The twisted cocycle $\Omega_{g}$ satisfies the following properties:

$$
\begin{gathered}
\Omega_{g}\left(y^{1} \otimes \cdots \otimes y^{l(g)}\right)=1 \\
\Omega_{g}\left(y^{1} \otimes \cdots \otimes c \otimes \cdots \otimes y^{l(g)}\right)=0,
\end{gathered}
$$

when $c$ is a constant function.

Proof. A straightforward check.

We remark that the expression (3) of $\Omega_{g}$ depends on the choices of coordinates $y^{1}, \ldots, y^{l(g)}$. This makes $\Omega_{g}$ in general not invariant with respect to the conjugate action. However, we have the following property.

Proposition 3.2. Let $C(g)$ be the centralizer subgroup of $g$, which acts on $N^{g}$. If the $C(g)$-action on $N^{g}$ is diagonalizable 2 there is a natural construction of $\Omega_{g}$ such that

$$
h\left(\Omega_{g}\right)=\operatorname{det}\left(\left.h\right|_{N^{g}}\right) \Omega_{g}, \quad h \in C(g) .
$$

Proof. As a $C(g)$-action on $N^{g}$ is diagonalizable and $g$ commutes with elements in $C(g), g$ and the $C(g)$-action on $N^{g}$ can be diagonalized simultaneously. Therefore, we can find coordinates $y^{1}, \ldots, y^{l(g)}$ on $N^{g}$, which are eigenfunctions of $g$ and the $C(g)$-action. We define $\Omega_{g}$ using the coordinates $y^{i}$ as in Equation (3). In particular, $\tilde{y}^{i}=g^{i} y^{i}$, and $h\left(\tilde{y}^{i}\right)=h\left(g^{i} y^{i}\right)=g^{i} h^{i} y^{i}$, where $g^{i}$ and $h^{i}$ are eigenvalues

\footnotetext{
${ }^{2}$ By a diagonalizable action, we mean that a $C(g)$-action on $N^{g}$ splits into a direct sum of 1-dim or 2-dim representations of $C(g)$.
} 
of $g$ and the $h$-action on $y^{i}$. Plugging these expressions in the definition of $h\left(\Omega_{g}\right)$, we obtain the equation

$$
h\left(\Omega_{g}\right)=\operatorname{det}\left(\left.h\right|_{N^{g}}\right) \Omega_{g}, \quad h \in C(g) .
$$

There are two special cases that we know of in which the conditions assumed in Proposition 3.2 are satisfied:

(1) Group $G$ is abelian.

(2) The codimension $l(g)$ is 1 or 2 . $C(g)$ acts on $N^{g}$ by isometry. When $l(g)=1,2$, the isometry group of $N^{g}$ is abelian.

At the end of this subsection, we give an example of the twisted cyclic cocycle in a very special case.

Example 3.3. Let $V$ be $\mathbb{R}$, and let $G=\mathbb{Z} / 2 \mathbb{Z}=\{i d, e\}$ act on $\mathbb{R}$ by $e: x \mapsto-x$. In this case, $\Omega_{e} \in C^{1}\left(A, A_{e}\right)$ is defined to be

$$
\Omega_{e}(f)(x)=\frac{f(x)-f(-x)}{2 x} .
$$

The cohomology $H H^{\bullet}\left(A, A_{e}\right)$ is computed to be

$$
H H^{\bullet}\left(A, A_{e}\right)= \begin{cases}0 & \bullet \neq 1 \\ \mathbb{R} & \bullet=1\end{cases}
$$

where $H H^{1}\left(A, A_{e}\right)$ is generated by $\Omega_{e}$.

3.2. Inverse map of $L$. We use the twisted cocycle constructed in the previous step to obtain an inverse map of $L$ :

$$
T: \Gamma^{\infty}\left(\bigoplus_{g \in G} \wedge^{\bullet-l(g)} T V^{g} \otimes \wedge^{l(g)} N^{g}\right)^{G} \longrightarrow C^{\bullet}(\operatorname{Poly}(V) \rtimes G, \operatorname{Poly}(V) \rtimes G) .
$$

We define $T$ as a composition of two maps $T_{1}$ and $T_{2}$. The map $T_{1}$ is defined as

$$
T_{1}: \Gamma^{\infty}\left(\bigoplus_{g \in G} \wedge^{\bullet-l(g)} T V^{g} \otimes \wedge^{l(g)} N^{g}\right)^{G} \longrightarrow C^{\bullet}(A, \operatorname{Poly}(V) \rtimes G)^{G} .
$$

The map $T_{2}$ is the standard map constructed in the proof of the Eilenberg-Zilber theorem:

$T_{2}: C^{\bullet}(A, \operatorname{Poly}(V) \rtimes G)^{G} \subset C^{0}\left(G, C^{\bullet}(A, \operatorname{Poly}(V) \rtimes G)\right) \longrightarrow C^{\bullet}(A \rtimes G, \operatorname{Poly}(V) \rtimes G)$.

Step I. The map $T_{1}$ is a sum of the maps

$$
T_{1}^{g}: \Gamma^{\infty}\left(\wedge^{\bullet-l(g)} T V^{g} \otimes \wedge^{l(g)} N^{g}\right) \longrightarrow C^{\bullet}\left(A, A_{g}\right),
$$

which are defined as follows.

Given $\xi \in \Gamma^{\infty}\left(\wedge^{\bullet-l(g)} T V^{g} \otimes \wedge^{l(g)} N^{g}\right)$, we write $\xi$ to be $X \otimes \Lambda_{g}$, where $X \in$ $\Gamma^{\infty}\left(\wedge^{\bullet-l(g)} T V^{g}\right)$ and $\Lambda_{g} \in \Gamma^{\infty}\left(\wedge^{l(g)} N^{g}\right)$ is defined be

$$
\frac{\partial}{\partial x^{n-l(g)+1}} \wedge \cdots \wedge \frac{\partial}{\partial x^{n}} \text {. }
$$

We define

$$
T_{1}^{g}(\xi)=X \sharp \Omega_{g}, \quad \text { for any } \xi \in \Gamma^{\infty}\left(\wedge^{k-l(g)} T V^{g} \otimes \wedge^{l(g)} N^{g}\right),
$$


where we view $X$ as a multi-differential operator on $\operatorname{Poly}(V)$ for $X \in \wedge^{k-l(g)} T V^{g}$ and $X \sharp \Omega_{g}\left(f_{1}, \ldots, f_{k}\right)$ equal to

$$
X\left(f_{1}, \ldots, f_{k-l(g)}\right) \Omega_{g}\left(f_{k-l(g)+1}, \ldots, f_{k}\right) .
$$

When the $C(g)$-action on $N^{g}$ is diagonizable, as is explained in Proposition 3.2. we have

$$
T_{1}^{g}(h(\xi))=h\left(T_{1}^{h^{-1} g h}(\xi)\right), \quad \forall h \in G .
$$

We define $T_{1}$ to be the sum of $T_{1}^{g}$. The restriction of $T_{1}$ to the $G$-invariant sections gives the desired map

$$
T_{1}: \Gamma^{\infty}\left(\bigoplus_{g \in G} \wedge^{\bullet-l(g)} T V^{g} \otimes \wedge^{l(g)} N^{g}\right)^{G} \longrightarrow C^{\bullet}(A, \operatorname{Poly}(V) \rtimes G)^{G} .
$$

When the $C(g)$-action on $N^{g}$ is not diagonalizable, then the image of the above $T_{1}$ map is not always $G$-invariant. Therefore, we need to replace $T_{1}$ by

$$
\frac{1}{|G|} \sum_{h} h\left(T_{1}(\xi)\right) \text {. }
$$

Step II. We now explain the construction of $T_{2}$, which is standard in the EilenbergZilber theorem. Given $\Phi \in C^{k}(A, \operatorname{Poly}(V) \rtimes G)$,

$$
T_{2}(\Phi)\left(a_{1} U_{g_{1}}, \ldots, a_{k} U_{g_{k}}\right)=\Phi\left(a_{1}, \ldots, g_{1} \cdots g_{k-1}\left(a_{k}\right)\right) U_{g_{1} \cdots g_{k}} .
$$

By this Lemma 3.1, we have the following proposition for the map $L_{2}$.

Proposition 3.4. Given $\xi \in \Gamma^{\infty}\left(\bigoplus_{g} \wedge^{k-l(g)} T V^{g} \otimes \wedge^{l(g)} N^{g}\right)^{G}$, we write $\xi=$ $\sum_{g} X_{g} \otimes \Lambda_{g}$, where $\Lambda_{g}$ is defined as in Equation (4).

The composition map $L_{2} \circ T_{1}$ satisfies

$$
L_{2}\left(T_{1}(\xi)\right)=\sum_{g} X_{g} \otimes \Lambda_{g}=\xi .
$$

Proof. As $L_{2}$ is $G$-equivariant and $\xi$ is $G$-invariant, we compute $L_{2}\left(T_{1}(\xi)\right)(x)$ as follows: $L_{2}\left(T_{1}(\xi)\right)(x)=$

$$
\begin{aligned}
= & \sum_{i_{1}, \ldots, i_{k}} T_{1}(\xi)\left(\left(x_{1}-x\right)^{i_{1}} \cdots\left(x_{k}-x\right)^{i_{k}}\right) \frac{\partial}{\partial x^{i_{1}}} \wedge \cdots \wedge \frac{\partial}{\partial x^{i_{k}}} \\
= & \sum_{g} \sum_{i_{1}, \ldots, i_{k}} T_{1}\left(X_{g} \otimes \Lambda_{g}\right)\left(\left(x_{1}-x\right)^{i_{1}} \cdots\left(x_{k}-x\right)^{i_{k}}\right) \frac{\partial}{\partial x^{i_{1}}} \wedge \cdots \wedge \frac{\partial}{\partial x^{i_{k}}} \\
= & \sum_{g} \sum_{i_{1}, \ldots, i_{k}} X_{g}\left(\left(x_{1}-x\right)^{i_{1}}, \ldots,\left(x_{k-l(g)}-x\right)^{i_{k-l(g)}}\right) \\
& \times \Omega_{g}\left(\left(x_{k-l(g)+1}-x\right)^{i_{k-l(g)+1}}, \ldots,\left(x_{k}-x\right)^{i_{k}}\right) \frac{\partial}{\partial x^{i_{1}}} \wedge \cdots \wedge \frac{\partial}{\partial x^{i_{k}}} \\
= & \sum_{g} \sum_{i_{1}, \ldots, i_{k}} X_{g}\left(\left(x_{1}-x\right)^{i_{1}}, \ldots,\left(x_{k-l(g)}-x\right)^{i_{k-l(g)}}\right) \frac{\partial}{\partial x^{i_{1}}} \wedge \cdots \wedge \frac{\partial}{\partial x^{i_{k-l(g)}}} \\
& \otimes \Omega_{g}\left(\left(x_{k-l(g)+1}-x\right)^{i_{k-l(g)+1}}, \ldots,\left(x_{k}-x\right)^{i_{k}}\right) \frac{\partial}{\partial x^{i_{k-l(g)+1}}} \wedge \cdots \wedge \frac{\partial}{\partial x^{i_{k}}} \\
= & \sum_{g} X_{g} \otimes \Lambda_{g} .
\end{aligned}
$$

In the last equality, we used Lemma 3.1, which shows $\Lambda_{g}$ and $\Omega_{g}$ have the same values on linear functions. 
We define $T=T_{2} \circ T_{1}$, and have the following theorem.

Theorem 3.5. The map $T$ is a quasi-isomorphism. In particular, $L \circ T=i d$.

Proof. We notice that $L_{1}\left(T_{2}\right)=i d$ on $C^{\bullet}(A ; A \rtimes G)^{G}$, and therefore have

$$
L \circ T(\xi)=L_{3}\left(L_{2}\left(L_{1}\left(T_{2}\left(T_{1}(\xi)\right)\right)\right)\right)=L_{3}\left(L_{2}\left(T_{1}(\xi)\right)\right),
$$

which is equal to $\xi$ by Proposition 3.4 .

3.3. The case of a smooth manifold. In this subsection, we discuss the extension of the construction of $T$ to general manifolds, which is again a composition of $T_{2}$ and $T_{1}$.

The map $T_{2}$ generalizes to the manifold case directly because it is purely algebraic. The same formula as Equation (5) defines a map

$$
T_{2}:\left(C^{\bullet}\left(C^{\infty}(M), C^{\infty}(M) \rtimes G\right)\right)^{G} \longrightarrow C^{\bullet}\left(C^{\infty}(M) \rtimes G, C^{\infty}(M) \rtimes G\right) .
$$

To define $T_{1}$, we consider a $g$-invariant tubular neighborhood $\mathcal{M}^{g}$ of $M^{g}$. The neighborhood $\mathcal{M}^{g}$ is a fiber bundle over $M^{g}$, and we fix a $G$-invariant Ehresmann connection on $\mathcal{M}^{g}$. Furthermore, we choose a cutoff function $\rho_{g}$ on $\mathcal{M}^{g}$ which is equal to 1 on a $g$-invariant neighborhood of $M^{g}$ and vanishes outside $\mathcal{M}^{g}$. Given a section $\xi_{g}=X_{g} \otimes \Lambda_{g}$ of $\wedge^{\bullet-l(g)} T M^{g} \otimes \wedge^{l(g)} N^{g}$ to $\mathcal{M}^{g}$, we use the Ehresmann connection to extend $X_{g}$ to a multi-vector field $\tilde{X}_{g}$ on $\mathcal{M}^{g}$, and we define $\Omega_{g}$ to be a linear map on $C^{\infty}\left(\mathcal{M}^{g}\right)$ by the same formula as Equation (3).

We define a cochain map

$$
T_{1}: \Gamma^{\infty}\left(\bigoplus_{g} \wedge^{k-l(g)} T M^{g} \otimes \wedge^{l(g)} N^{g}\right)^{G} \longrightarrow C^{k}\left(C^{\infty}(M) ; C^{\infty}(M) \rtimes G\right)^{G}
$$

by

$$
T_{1}(\xi)\left(f_{1}, \ldots, f_{k}\right)=\sum_{g} \rho_{g} \tilde{X}_{g}\left(f_{1}, \ldots, f_{k-l(g)}\right) \Omega_{g}\left(f_{k-l(g)+1}, \ldots, f_{k}\right) U_{g},
$$

for $\xi=\sum_{g} X_{g} \otimes \Lambda_{g}$

Again, we can easily compute that $L \circ T=i d$. Therefore, since $L$ is a quasiisomorphism proved in [13, $T$ is also a quasi-isomorphism.

Remark 3.6. The definition of $T_{1}$ depends on a choice of the normal bundle $N^{g}$, the $G$-invariant Ehresmann connection, and the cutoff function. Therefore, $T$ is not a canonical map. However, we notice that at any $x \in M^{g}$, inside $T_{x} M$, there is a canonical complementary subspace to $T_{x} M^{g} \subset T_{x} M$ determined by the representation of $g$ on $T_{x} M$ independent of the choices of the metrics. Therefore, it is easy to check ( $c f$. Proposition 3.4) that when restricted to the $\infty$-jets of $M^{g}$, the map $T$ is independent of all the choices.

\section{Gerstenhaber bracket}

In this section, we compute the Gerstenhaber bracket on the Hochschild cohomology of the algebra $C^{\infty}(M) \rtimes G$. Since all the computations and constructions are local with respect to the orbifold $M / G$, it is sufficient to work out everything locally on a vector space. Because the Gerstenhaber bracket on $C^{\bullet}(\operatorname{Poly}(V) \rtimes G, \operatorname{Poly}(V) \rtimes G)$ is the commutator of the pre-Lie product, we mainly work on understanding the pre-Lie product, and we state the results for the Gerstenhaber bracket. 
4.1. Geometric description. Let $\xi \in \Gamma^{\infty}\left(\bigoplus_{\alpha \in G} \wedge^{k-l(\alpha)} T V^{\alpha} \otimes \wedge^{l(\alpha)} N^{\alpha}\right)^{G}$ and $\eta \in \Gamma^{\infty}\left(\bigoplus_{\beta \in G} \wedge^{l-l(\beta)} T V^{\beta} \otimes \wedge^{l(\beta)} N^{\beta}\right)^{G}$. We compute the pre-Lie product between $T(\xi)$ and $T(\eta)$ by $L(T(\xi) \circ T(\eta))$. We write $\xi$ as the sum of $\xi_{\alpha}=X_{\alpha} \otimes \Lambda_{\alpha}$, and $\eta$ as the sum of $\eta_{\beta}=Y_{\beta} \otimes \Lambda_{\beta}$, with $X_{\alpha} \in \Gamma^{\infty}\left(\wedge^{k-l(\alpha)} T V^{\alpha}\right), Y_{\beta} \in \Gamma^{\infty}\left(\wedge^{l-l(\beta)} T V^{\beta}\right)$, $\Lambda_{\alpha} \in \Gamma^{\infty}\left(\wedge^{l(\alpha)} N^{\alpha}\right), \Lambda_{\beta} \in \Gamma^{\infty}\left(\wedge^{l(\beta)} N^{\beta}\right)$. We compute the Gerstenhaber bracket between $T(\xi)$ and $T(\eta)$ by $L([T(\xi), T(\eta)])$ using the information of the quasiisomorphisms $L$ and $T$ introduced in Sections 2 and 3.

Step I. We compute $L_{1}(T(\xi) \circ T(\eta)) \in C^{k+l-1}(\operatorname{Poly}(V), \operatorname{Poly}(V) \rtimes G)$ first. As $\xi$ and $\eta$ are both $G$-invariant, the cocycles $T_{1}(\xi), T_{1}(\eta) \in C^{\bullet}(\operatorname{Poly}(V), \operatorname{Poly}(V) \rtimes G)$ are also $G$-invariant, and therefore the averaging in the definition of $L_{1}$ is not necessary. Our computations yield:

$$
\begin{aligned}
& L_{1}(T(X) \circ T(Y))\left(f_{1}, \ldots, f_{k+l-1}\right) \\
&= \sum_{s}(-1)^{(s-1)(l-1)} T(\xi)\left(f_{1}, \ldots, f_{s}, T(\eta)\left(f_{s+1}, \ldots, f_{s+l}\right), f_{s+l+1}, \ldots, f_{k+l-1}\right) \\
&= \sum_{s}(-1)^{(s-1)(l-1)} T\left(\sum_{\alpha} \xi_{\alpha}\right)\left(f_{1}, \ldots, f_{s}, T\left(\sum_{\beta} \eta_{\beta}\right)\left(f_{s+1}, \ldots, f_{s+l}\right), f_{s+l+1}, f_{k+l-1}\right) \\
&= \sum_{\alpha} \sum_{\beta} \sum_{s}(-1)^{(s-1)(l-1)} T\left(\xi_{\alpha}\right)\left(f_{1}, \ldots, f_{s}, T\left(\eta_{\beta}\right)\left(f_{s+1}, \ldots, f_{s+l}\right),\right. \\
&= \sum_{g} \sum_{\alpha \beta=g} \sum_{s}(-1)^{(s-1)(l-1)} T_{1}^{\alpha}\left(\xi_{\alpha}\right)\left(f_{1}, \ldots, f_{s}, T_{1}^{\beta}\left(\eta_{\beta}\right)\left(f_{s+1}, \ldots, f_{s+l}\right),\right. \\
&\left.\beta\left(f_{s+l+1}\right), \ldots, \beta\left(f_{k+l-1}\right)\right) U_{g} .
\end{aligned}
$$

Step II. We compute $L_{2}^{g}\left(L_{1}(T(\xi) \circ T(\eta))\right)(x)$ by applying $L_{2}^{g}$ to the computation in the previous step:

(6)

$$
\begin{gathered}
\frac{1}{|G|^{2}} \sum_{i_{1}, \ldots, i_{k+l-1}} \sum_{\alpha \beta=g, h_{1}, h_{2} \in G} \sum_{s}(-1)^{(s-1)(l-1)} h_{1}\left(T_{1}^{\alpha}\left(\xi_{\alpha}\right)\right)\left(\left(x_{1}-x\right)^{i_{1}}, \ldots,\left(x_{s}-x\right)^{i_{s}},\right. \\
h_{2}\left(T_{1}^{\beta}\left(\eta_{\beta}\right)\right)\left(\left(x_{s+1}-x\right)^{i_{s+1}}, \ldots,\left(x_{s+l}-x\right)^{i_{x+l}}\right), \beta\left(\left(x_{s+l+1}-x\right)^{i_{s+l+1}}\right), \ldots, \\
\left.\beta\left(\left(x_{k+l-1}-x\right)^{i_{k+l-1}}\right)\right) \frac{\partial}{\partial x^{i_{1}}} \wedge \cdots \wedge \frac{\partial}{\partial x^{i_{k+l-1}}} .
\end{gathered}
$$

We look at the term $T_{1}^{\beta}\left(\eta_{\beta}\right)\left(\left(x_{s+1}-x\right)^{i_{s+1}}, \ldots,\left(x_{s+l}-x\right)^{i_{s+l}}\right)$. Using the expression $\eta_{\beta}=X_{\beta} \otimes \Lambda_{\beta}$, we have

$$
\begin{aligned}
& T_{1}^{\beta}\left(\eta_{\beta}\right)\left(\left(x_{s+1}-x\right)^{i_{s+1}}, \ldots,\left(x_{s+l}-x\right)^{i_{s+l}}\right) \\
= & X_{\beta} \sharp \Omega_{\beta}\left(\left(x_{s+1}-x\right)^{i_{s+1}}, \ldots,\left(x_{s+l}-x\right)^{i_{s+l}}\right) \\
= & X_{\beta}\left(\left(x_{s+1}-x\right)^{i_{s+1}}, \ldots,\left(x_{s+l-l(\beta)}-x\right)^{i_{s+l-l(\beta)}}\right) \\
\times & \Lambda_{\beta}\left(\left(x_{s+l-l(\beta)}-x\right)^{i_{s+l-l(\beta)+1}}, \ldots,\left(x_{s+l}-x\right)^{i_{s+l}}\right) \\
= & \eta_{\beta}\left(\left(x_{s+1}-x\right)^{i_{s+1}}, \ldots,\left(x_{s+l}-x\right)^{i_{s+l}}\right) .
\end{aligned}
$$

In the second equality of the above equation, we used Lemma 3.1, which stated that $\Omega_{\beta}$ and $\Lambda_{\beta}$ agree on linear functions. 
Substituting the above expression of the $T_{1}^{\beta}\left(\eta_{\beta}\right)$ into Equation (6), as $\eta$ is $G$ invariant, we have

$$
\begin{aligned}
& L_{2}^{g}\left(T\left(\xi_{\alpha}\right) \circ T\left(\eta_{\beta}\right)\right) \\
&=\frac{1}{|G|} \sum_{h} \sum_{i_{1}, \ldots, i_{k+l-1}} \sum_{s}(-1)^{(s-1)(l-1)} h\left(T_{1}^{\alpha}\left(\xi_{\alpha}\right)\right)\left(\left(x_{1}-x\right)^{i_{1}}, \ldots,\left(x_{s}-x\right)^{i_{s}},\right. \\
& \eta_{\beta}\left(\left(x_{s+1}-x\right)^{i_{s+1}}, \ldots,\left(x_{s+l}-x\right)^{i_{s+l}}\right), \beta\left(\left(x_{s+l+1}-x\right)^{i_{s+l+1}}\right), \ldots, \\
&\left.\beta\left(\left(x_{k+l-1}-x\right)^{i_{k+l-1}}\right)\right) \frac{\partial}{\partial x^{i_{1}}} \wedge \cdots \wedge \frac{\partial}{\partial x^{i_{k+l-1}}} .
\end{aligned}
$$

We discuss several properties of $L_{2}^{g}\left(T\left(\xi_{\alpha}\right) \circ T\left(\eta_{\beta}\right)\right)$.

Lemma 4.1. The restriction of $L\left(T\left(\xi_{\alpha}\right) \circ T\left(\eta_{\beta}\right)\right)$ to $V^{\alpha} \cap V^{\beta}$ is

$$
\begin{gathered}
\sum_{i_{1}, \ldots, i_{k+l-1}} \sum_{s}(-1)^{(s-1)(l-1)} \xi_{\alpha}\left(\left(x_{1}-x\right)^{i_{1}}, \ldots,\left(x_{s}-x\right)^{i_{s}},\right. \\
\eta_{\beta}\left(\left(x_{s+1}-x\right)^{i_{s+1}}, \ldots,\left(x_{s+l}-x\right)^{i_{s+l}}\right), \beta\left(\left(x_{s+l+1}-x\right)^{i_{s+l+1}}\right), \ldots, \\
\left.\beta\left(\left(x_{k+l-1}-x\right)^{i_{k+l-1}}\right)\right) \frac{\partial}{\partial x^{i_{1}}} \wedge \cdots \wedge \frac{\partial}{\partial x^{i_{k+l-1}}} .
\end{gathered}
$$

Proof. Because $T_{1}\left(\xi_{\alpha}\right) \in C^{k}\left(\operatorname{Poly}(V) ; \operatorname{Poly}(V)_{\alpha}\right)$ and $T_{1}\left(\eta_{\beta}\right) \in C^{l}(\operatorname{Poly}(V)$; $\left.\operatorname{Poly}(V)_{\beta}\right)$, the cocycle $T_{1}\left(\xi_{\alpha}\right) \circ T_{1}\left(\eta_{\beta}\right)$ is in $C^{k+l-1}\left(\operatorname{Poly}(V) ; \operatorname{Poly}(V)_{\alpha \beta}\right)$. Therefore, the restriction of $L\left(T\left(\xi_{\alpha}\right) \circ T\left(\eta_{\beta}\right)\right)$ to $V^{\alpha} \cap V^{\beta}$ is the same as the restriction of $\left.L_{2}^{\alpha \beta}\left(T_{1}\left(\xi_{\alpha}\right) \circ T_{1}\left(\eta_{\beta}\right)\right)\right)$ to $V^{\alpha} \cap V^{\beta}$.

We observe that the restriction of $\Omega_{\alpha}\left(f_{1}, \ldots, f_{l(\alpha)}\right)$ (and $\Omega_{\beta}(\cdots)$ ) to $V^{\alpha}$ (and $V^{\beta}$ ) is the same as $\Lambda_{\alpha}\left(f_{1}, \ldots, f_{l(\alpha)}\right)$ (and $\Lambda_{\beta}(\cdots)$ ) as we set all the variables along the normal direction of $V^{\alpha}$ equal to 0 . Using this property and the $G$-invariance of $\xi$, we have that the restriction of

$$
\begin{aligned}
& T_{1}\left(\xi_{\alpha}\right)\left(\left(x_{1}-x\right)^{i_{1}}, \ldots,\left(x_{s}-x\right)^{i_{s}}, T_{1}\left(\eta_{\beta}\right)\left(\left(x_{s+1}-x\right)^{i_{s+1}}, \ldots,\left(x_{s+l}-x\right)^{i_{s+l}}\right),\right. \\
& \left.\beta\left(\left(x_{s+l+1}-x\right)^{i_{s+l+1}}\right), \ldots, \beta\left(\left(x_{k+l-1}-x\right)^{i_{k+l-1}}\right)\right)
\end{aligned}
$$

to $V^{\alpha} \cap V^{\beta}$ is the same as

$$
\begin{aligned}
& \xi_{\alpha}\left(\left(x_{1}-x\right)^{i_{1}}, \ldots,\left(x_{s}-x\right)^{i_{s}}, \eta_{\beta}\left(\left(x_{s+1}-x\right)^{i_{s+1}}, \ldots,\left(x_{s+l}-x\right)^{i_{s+l}}\right),\right. \\
& \left.\beta\left(\left(x_{s+l+1}-x\right)^{i_{s+l+1}}\right), \ldots, \beta\left(\left(x_{k+l-1}-x\right)^{i_{k+l-1}}\right)\right) .
\end{aligned}
$$

Inspired by the results of Lemma 4.1, we introduce the following definition.

Definition 4.2. Let $V$ be a vector space with a linear endomorphism $\gamma$. For all $\xi \in \wedge^{k} T V, \eta \in \wedge^{l} T V$, the $\gamma$-twisted pre-Lie product $\xi \circ_{\gamma} \eta$ is defined to be

$$
\begin{gathered}
\sum_{i_{1}, \ldots, i_{k+l-1}} \sum_{s}(-1)^{(s-1)(l-1)} \xi\left(\left(x_{1}-x\right)^{i_{1}}, \ldots,\left(x_{s}-x\right)^{i_{s}},\right. \\
\eta\left(\left(x_{s+1}-x\right)^{i_{s+1}}, \ldots\left(x_{s+l}-x\right)^{i_{s+l}}\right), \gamma\left(\left(x_{s+l+1}-x\right)^{i_{s+l+1}}\right), \ldots, \\
\left.\gamma\left(\left(x_{k+l-1}-x\right)^{i_{k+l-1}}\right)\right)(x) \frac{\partial}{\partial x^{i_{1}}} \wedge \cdots \wedge \frac{\partial}{\partial x^{i_{k+l-1}}} .
\end{gathered}
$$


Step III. In the following, we look at the $\alpha \beta$ component of the Gerstenhaber bracket in the case when $V^{\alpha} \cap V^{\beta}=V^{\alpha \beta}$.

Lemma 4.3. Assume $V^{\alpha} \cap V^{\beta}=V^{\alpha \beta}$. If $\xi_{\alpha}=X_{\alpha} \otimes \Lambda_{\alpha}$ contains directions along $N^{\beta}$, then the contribution of $\xi_{\alpha}$ and $\eta_{\beta}$ to the $\alpha \beta$ component of $L\left(T\left(\xi_{\alpha}\right) \circ T\left(\eta_{\beta}\right)\right)$ vanishes.

Proof. Firstly, as $V^{\alpha \beta}=V^{\alpha} \cap V^{\beta}$, we can apply Lemma 3.1 to compute $L\left(T\left(\xi_{\alpha}\right) \circ\right.$ $\left.T\left(\eta_{\beta}\right)\right)$ using the twisted pre-Lie product between $\xi_{\alpha}$ and $\eta_{\beta}$.

Secondly, we look at the term $\eta_{\beta}(\cdots)$ in $X_{\alpha} \circ_{\alpha} \eta_{\beta}$ at $g=\alpha \beta$. It contains derivations along all the directions of $N^{\beta}$.

Thirdly, by Lemma 3.1 if the component of $L\left(T\left(\xi_{\alpha}\right) \circ T\left(\eta_{\beta}\right)\right)$ at $\frac{\partial}{\partial x^{i_{1}}} \wedge \cdots \wedge$ $\frac{\partial}{\partial x^{i_{k+l-1}}}$ does not vanish, we must have at least one of $\left(x_{1}-x\right)^{i_{1}}, \ldots,\left(x_{s}-x\right)^{i_{s}}$, $\eta_{\beta}\left(\left(x_{s+1}-x\right)^{i_{s+1}}, \ldots,\left(x_{s+l}-x\right)^{i_{s+l}}\right), \beta\left(\left(x_{s+l+1}-x\right)^{i_{s+l+1}}\right), \ldots, \beta\left(\left(x_{k+l-1}-x\right)^{i_{k+l-1}}\right)$ which is supported along the $N^{\beta}$ direction because $X_{\alpha} \otimes \Lambda_{\alpha}$ contains derivations along $N^{\beta}$. Furthermore, we notice that $\eta_{\beta}\left(\left(x_{s+1}-x\right)^{i_{s+1}}, \ldots,\left(x_{s+l}-x\right)^{i_{s+l}}\right)$ lies along the $V^{\beta}$ direction. This implies that at least one of $\left(x_{1}-x\right)^{i_{1}}, \ldots,\left(x_{s}-\right.$ $x)^{i_{s}}, \beta\left(\left(x_{s+l+1}-x\right)^{i_{s+l+1}}\right), \ldots, \beta\left(\left(x_{k+l-1}-x\right)^{i_{k+l-1}}\right)$ must be along the $N^{\beta}$ direction. Noticing that the action of $\beta$ on $V$ preserves the decomposition $V=$ $V^{\beta} \oplus N^{\beta}$, we conclude that at least one of $\left(x_{1}-x\right)^{i_{1}}, \ldots,\left(x_{s}-x\right)^{i_{s}},\left(x_{s+l+1}-\right.$ $x)^{i_{s+l+1}}, \ldots,\left(x_{k+l-1}-x\right)^{i_{k+l-1}}$ is along $N^{\beta}$.

Summarizing the above observations, we see that $T\left(X_{\alpha} \otimes \Lambda_{\alpha}\right) \circ T\left(Y_{\beta} \otimes \Lambda_{\beta}\right)$ contains too many derivations along $N^{\beta}$ since in the above two parts we have a total of more than $l(\beta)$ many derivations along the $N^{\beta}$ direction, which is of dimension $l(\beta)$. We conclude that

$$
L\left(T\left(X_{\alpha} \otimes \Lambda_{\alpha}\right) \circ T\left(Y_{\beta} \otimes \Lambda_{\beta}\right)\right)=L\left(T\left(\xi_{\alpha}\right) \circ T\left(\eta_{\beta}\right)\right)=0 .
$$

Lemma 4.3 shows that to compute the $\alpha \beta$ component of $L\left(T\left(\xi_{\alpha}\right) \circ T\left(\eta_{\beta}\right)\right)$ we can assume that $\xi_{\alpha}$ is contained in the $V^{\beta}$ direction. Therefore, there is no need to consider the $\beta$-action on $\left(x_{s+l+1}-x\right)^{i_{s+l+1}}, \ldots,\left(x_{k+l-1}-x\right)^{i_{k+l-1}}$ in Equation (7), which concerns $L\left(T\left(\xi_{\alpha}\right) \circ T\left(\eta_{\beta}\right)\right)$. In this case, the twisted pre-Lie product between $\xi_{\alpha}$ and $\eta_{\beta}$ is reduced to

$$
\begin{aligned}
& \sum_{i_{1}, \ldots, i_{k+l-1}} \sum_{s}(-1)^{(s-1)(l-1)} \xi_{\alpha}\left(\left(x_{1}-x\right)^{i_{1}}, \ldots,\left(x_{s}-x\right)^{i_{s}},\right. \\
& \quad \eta_{\beta}\left(\left(x_{s+1}-x\right)^{i_{s+1}}, \ldots\left(x_{s+l}-x\right)^{i_{s+l}}\right), \\
& \left.\quad\left(x_{s+l+1}-x\right)^{i_{s+l+1}}, \ldots,\left(x_{k+l-1}-x\right)^{i_{k+l-1}}\right) \frac{\partial}{\partial x^{i_{1}}} \wedge \cdots \wedge \frac{\partial}{\partial x^{i_{k+l-1}}} .
\end{aligned}
$$

This is the standard pre-Lie product $\xi_{\alpha} \circ \eta_{\beta}$ between $\xi_{\alpha}$ and $\eta_{\beta}$.

We summarize the above computation into the following theorem 3

Theorem 4.4. Consider

$$
\begin{aligned}
& \xi=\sum_{\alpha} \xi_{\alpha} \in \Gamma^{\infty}\left(\bigoplus_{\alpha} \wedge^{k-l(\alpha)} T V^{\alpha} \otimes \wedge^{l(\alpha)} N^{\alpha}\right)^{G}, \\
& \eta=\sum_{\beta} \eta_{\beta} \in \Gamma^{\infty}\left(\bigoplus_{\beta} \wedge^{l-l(\beta)} T V^{\beta} \otimes \wedge^{l(\beta)} N^{\beta}\right)^{G} .
\end{aligned}
$$

\footnotetext{
${ }^{3}$ A theorem similar to the one that follows was stated in the first version of [1, but the proof there contained a crucial gap.
} 
If $V^{\alpha} \cap V^{\beta}=V^{\alpha \beta}$, then the contribution of $\xi_{\alpha}$ and $\eta_{\beta}$ to the $\alpha \beta$ component of $L\left(T\left(\xi_{\alpha}\right) \circ T\left(\eta_{\beta}\right)\right)$ is

$$
\operatorname{pr}^{\alpha \beta}\left(\left.\xi_{\alpha} \circ \eta_{\beta}\right|_{V^{\alpha \beta}}\right),
$$

where $\circ$ in the above formula is the standard pre-Lie product on $V$.

Proof. The proof is straightforward from Lemma 4.3 .

We discuss a special but important case that $V^{\alpha} \cap V^{\beta}=V^{\alpha \beta}$ holds true. The following lemma is well known, and we include its proof for the reader's convenience.

Lemma 4.5. For $\alpha, \beta \in G$, the condition $V^{\alpha}+V^{\beta}=V$ is equivalent to the equality $l(\alpha)+l(\beta)=l(\alpha \beta)$, and when $V^{\alpha}+V^{\beta}=V, V^{\alpha} \cap V^{\beta}=V^{\alpha \beta}$.

Proof. As $\operatorname{dim}\left(V^{\alpha}\right)+\operatorname{dim}\left(V^{\beta}\right)=\operatorname{dim}\left(V^{\alpha}+V^{\beta}\right)+\operatorname{dim}\left(V^{\alpha} \cap V^{\beta}\right)$, we have that

$$
\begin{aligned}
l(\alpha)+l(\beta) & =\operatorname{dim}(V)-\operatorname{dim}\left(V^{\alpha}\right)+\operatorname{dim}(V)-\operatorname{dim}\left(V^{\beta}\right) \\
& =\operatorname{dim}(V)-\operatorname{dim}\left(V^{\alpha}+V^{\beta}\right)+\operatorname{dim}(V)-\operatorname{dim}\left(V^{\alpha} \cap V^{\beta}\right) \\
& \geq \operatorname{dim}(V)-\operatorname{dim}\left(V^{\alpha}+V^{\beta}\right)+\operatorname{dim}(V)-\operatorname{dim}\left(V^{\alpha \beta}\right) \\
& =\operatorname{dim}(V)-\operatorname{dim}\left(V^{\alpha}+V^{\beta}\right)+l(\alpha \beta),
\end{aligned}
$$

where we have used the fact that $V^{\alpha} \cap V^{\beta} \subset V^{\alpha \beta}$. Therefore, $l(\alpha)+l(\beta)=l(\alpha \beta)$ implies that $V=V^{\alpha}+V^{\beta}$.

On the other hand, assume that $V^{\alpha}+V^{\beta}=V$ and let $\langle$,$\rangle be a G$-invariant metric on $V$. For any $v \in V^{\alpha \beta}$, we have $\alpha \beta(v)=v$, and accordingly $\beta(v)=\alpha^{-1}(v)$, and $\beta(v)-v=\alpha^{-1}(v)-v$. Furthermore, as the metric $\langle$,$\rangle is G$-invariant, we see that $\beta(v)-v$ is orthogonal to $V^{\beta}$ with respect to the metric $\langle$,$\rangle and \alpha^{-1}(v)-v$ is orthogonal to $V^{\alpha}$. Therefore $\beta(v)-v=\alpha^{-1}(v)-v$ is orthogonal to $V^{\alpha}+V^{\beta}$, which is equal to $V$ by the assumption. This implies that $v$ must belong to $V^{\alpha} \cap V^{\beta}$, and we have $V^{\alpha} \cap V^{\beta}=V^{\alpha \beta}$. This together with the above equations implies that

$$
l(\alpha)+l(\beta)=\operatorname{dim}(V)-\operatorname{dim}\left(V^{\alpha}+V^{\beta}\right)+\operatorname{dim}(V)-\operatorname{dim}\left(V^{\alpha \beta}\right)=l(\alpha \beta) .
$$

Example 4.6. We consider the set $S$ of all $g \in G$ such that $l(g)=2$. The set $S$ is invariant under conjugation as $l(g)$ is invariant under conjugate action. We consider $\pi=\sum_{g \in S} \pi_{g} \in \Gamma^{\infty}\left(\bigoplus_{g \in S} \wedge^{2} N^{g}\right)^{G}$ and $\phi_{i d} \in \Gamma^{\infty}\left(\wedge^{\bullet} V\right)^{G}$, which is supported at the identity component.

For $\pi$ and $\phi_{i d}$, the conditions of Theorem 4.4 are satisfied. Therefore, we have

$$
L\left(T(\pi) \circ T\left(\phi_{i d}\right)\right)=\sum_{g \in S} p r^{g}\left(\left.\pi_{g} \circ \phi_{i d}\right|_{V^{g}}\right)
$$

and

$$
L\left(\left[T(\pi), T\left(\phi_{i d}\right)\right]\right)=\sum_{g \in S} \operatorname{pr}^{g}\left(\left.\left[\pi_{g}, \phi_{i d}\right]\right|_{V^{g}}\right) .
$$

4.2. Abelian group action. In the following, we discuss the special case when $G$ is abelian. Under this assumption, we obtain a more explicit description of the twisted Schouten-Nijenhuis bracket. As $G$ is abelian, the $G$-action on $V$ is decomposed into a direct sum of 1 and 2 dimensional irreducible representations of $G$, and we have global well-defined coordinate functions, which are eigenvectors for all $g$-actions. In particular, the conditions of Proposition 3.2 are satisfied and the map $T_{1}$ in Section 3.2 without averaging is already $G$-equivariant. 
Remark 4.7. Discussions in this subsection can be extended to the case where all elements in $(\alpha)$ (the set of elements in the same conjugacy class as $\alpha$ ) commute with all elements in $(\beta)$.

Lemma 4.8. Let

$$
\xi=\sum_{\alpha} X_{\alpha} \otimes \Lambda_{\alpha} \in \Gamma^{\infty}\left(\bigoplus_{\alpha} \wedge^{k-l(\alpha)} T V^{\alpha} \otimes \wedge^{l(\alpha)} N^{\alpha}\right)^{G}
$$

and

$$
\eta=\sum_{\beta} Y_{\beta} \otimes \Lambda_{\beta} \in \Gamma^{\infty}\left(\bigoplus_{\beta} \wedge^{l-l(\beta)} T V^{\beta} \otimes \wedge^{l(\beta)} N^{\beta}\right)^{G} .
$$

If $V^{\alpha}+V^{\beta} \neq V$ for all $\alpha, \beta$, then

$$
L(T(\xi) \circ T(\eta))=0 .
$$

Proof. Following the computations similar to those in Equation (6), we have that $L\left(T\left(\xi_{(\alpha)}\right) \circ T\left(\eta_{(\beta)}\right)\right)$ is equal to

$$
\begin{gathered}
\sum_{i_{1}, \ldots, i_{k+l-1}} \sum_{\alpha \beta=g} \sum_{s}(-1)^{(s-1)(l-1)} T_{1}^{\alpha}\left(\xi_{\alpha}\right)\left(\left(x_{1}-x\right)^{i_{1}}, \ldots,\left(x_{s}-x\right)^{i_{s}},\right. \\
T_{1}^{\beta}\left(\eta_{\beta}\right)\left(\left(x_{s+1}-x\right)^{i_{s+1}}, \ldots,\left(x_{s+l}-x\right)^{i_{x+l}}\right), \\
\left.\beta\left(\left(x_{s+l+1}-x\right)^{i_{s+l+1}}\right), \ldots, \beta\left(\left(x_{k+l-1}-x\right)^{i_{k+l-1}}\right)\right) \frac{\partial}{\partial x^{i_{1}}} \wedge \cdots \wedge \frac{\partial}{\partial x^{i_{k+l-1}}} .
\end{gathered}
$$

Since $V^{\alpha}+V^{\beta} \neq V$, then its normal directions $N^{\perp}=N^{\alpha} \cap N^{\beta}$ are nontrivial. We observe that $T\left(\xi_{\alpha}\right)$ contains all the derivation 4 along $N^{\alpha}$, and $T\left(\eta_{\beta}\right)$ contains all the derivations along $N^{\beta}$. As $\eta_{\beta}$ is a section of $\wedge^{l-l(\beta)} T V^{\beta} \otimes \wedge^{l(\beta)} N^{\beta}, T_{1}^{\beta}\left(\eta_{\beta}\right)\left(\left(x_{s+1}-\right.\right.$ $\left.x)^{i_{s+1}}, \ldots,\left(x_{s+l}-x\right)^{i_{s+l}}\right)$ is independent of $N^{\perp}$. Therefore, we see that to have a nonzero contribution in $L\left(T\left(\xi_{\alpha}\right) \circ T\left(\eta_{\beta}\right)\right)$, the coordinates $x^{i_{1}}, \ldots, x^{i_{s+l}}, \beta\left(x^{i_{s+l+1}}\right)$, $\ldots, \beta\left(x^{i_{k+l-1}}\right)$ must contain two copies of the variables along $N^{\perp}$ and one copy of the variables along $N^{\beta} / N^{\perp}$. However, because $i_{1}, \ldots, i_{k+l-1}$ are distinguished and $N^{\beta}$ is $\beta$-invariant, the coordinates $x^{i_{1}}, \ldots, x^{s+k-1}, \beta\left(x^{s+k}\right), \ldots, \beta\left(x^{i_{k+l-1}}\right)$ have at most one copy of the variables along the $N^{\beta}$ direction. There are not enough variables along the $N^{\perp}$ direction. This implies that $L\left(T\left(\xi_{(\alpha)}\right) \circ T\left(\eta_{(\beta)}\right)\right)$ vanishes.

By Lemma 4.8, we are reduced to considering the Gerstenhaber bracket in the case when $V^{\alpha}+V^{\beta}=V$, which by Lemma 4.5 implies $V^{\alpha} \cap V^{\beta}=V^{\alpha \beta}$. Therefore, we can use Theorem 4.4 to compute the Gerstenhaber bracket.

Lemma 4.9. Let $G$ be an abelian group. If $l-l(\beta) \geq 2$, then for $Y_{\beta} \in$ $\Gamma^{\infty}\left(\wedge^{l-l(\beta)} T N^{\alpha}\right)^{C(\beta)}$,

$$
L\left(T\left(\xi_{\alpha}\right) \circ T\left(\eta_{\beta}\right)\right)=0 .
$$

Proof. Because of Lemma 4.8 and Theorem 4.4 we can drop the $\beta$ twist in Equation (17). At the component $g=\alpha \beta$, we look at the number of derivations along the direction of $N^{\alpha}$. The component $T\left(\xi_{\alpha}\right)$ contributes $l(\alpha)$ and $T\left(Y_{\beta} \otimes \Lambda_{\beta}\right)$ contributes $l-l(\beta)$ number of derivations. Therefore, $T\left(\xi_{\alpha}\right) \circ T\left(Y_{\beta} \otimes \Lambda_{\beta}\right)$ contains at least the following number of derivations along $N^{\alpha}$ :

$$
l(\alpha)+(l-l(\beta))-1 \geq l(\alpha)+2-1 \geq l(\alpha)+1 .
$$

This implies the statement of this lemma, because $\operatorname{dim}\left(N^{\alpha}\right)=l(\alpha)$.

\footnotetext{
${ }^{4}$ Rigorously speaking, $\Omega_{\alpha}, \Omega_{\beta}$ are not derivations. Here, we use the word "derivation" loosely, because they behave like derivations on linear functions.
} 
In the case when $G$ is abelian, the expressions of the pre-Lie product and Gerstenhaber bracket in Theorem 4.4 can be simplified.

Theorem 4.10. Let

$$
\xi=\sum_{\alpha \in(\alpha)} X_{\alpha} \otimes \Lambda_{\alpha} \in \Gamma^{\infty}\left(\wedge^{k-l(\alpha)} T V^{\alpha} \otimes \wedge^{l(\alpha)} N^{\alpha}\right)^{C(\alpha)}
$$

and

$$
\eta=\sum_{\beta \in(\beta)} Y_{\beta} \otimes \Lambda_{\beta} \in \Gamma^{\infty}\left(\wedge^{l-l(\beta)} T V^{\beta} \otimes \wedge^{l(\beta)} N^{\beta}\right)^{C(\beta)} .
$$

Then the component of $L(T(\xi) \circ T(\eta))$ in

$$
\Gamma^{\infty}\left(\wedge^{k+l-l(\alpha)-1} T V^{\alpha \beta} \otimes \wedge^{l(\alpha)} N^{\alpha \beta}\right)^{C(\alpha \beta)}
$$

is computed as follows.

(1) When $V^{\alpha}+V^{\beta} \neq V$ for all $\alpha, \beta$, then $L(T(\xi) \circ T(\eta))=0$.

(2) When $V^{\alpha}+V^{\beta}=V$, as $G$ is abelian, we write $V=V^{\alpha \beta} \oplus N^{\alpha} \oplus N^{\beta}$, where $V^{\alpha \beta}$ is the invariant subspace of $\alpha \beta, N^{\alpha}$ is the subspace orthogonal to $V^{\alpha}$, and $N^{\beta}$ is the subspace orthogonal to $V^{\beta}$. In this case, $V^{\alpha}=V^{\alpha \beta} \oplus N^{\beta}$ and $V^{\beta}=V^{\alpha \beta} \oplus N^{\alpha}$. According to this decomposition, we write

$$
\begin{aligned}
& X_{\alpha}=\sum X_{\alpha}^{i_{1} \cdots i_{s}, p_{1} \cdots p_{k-l(\alpha)-s}} \frac{\partial}{\partial x^{i_{1}}} \wedge \cdots \wedge \frac{\partial}{\partial x^{i_{s}}} \wedge \frac{\partial}{\partial x^{p_{1}}} \wedge \cdots \wedge \frac{\partial}{\partial x^{p_{k-l(\alpha)-s}}} \\
& \in \Gamma^{\infty}\left(\wedge^{s} T V^{\alpha \beta}\right) \otimes \Gamma^{\infty}\left(\wedge^{k-l(\alpha)-s} T N^{\beta}\right), \\
& Y_{\beta}=\sum Y_{\beta}^{j_{1} \cdots j_{t}, q_{1} \cdots q_{l-l(\beta)-t}} \frac{\partial}{\partial x^{j_{1}}} \wedge \cdots \wedge \frac{\partial}{\partial x^{j_{t}}} \wedge \frac{\partial}{\partial x^{q_{1}}} \wedge \cdots \wedge \frac{\partial}{\partial x^{q_{l-l(\beta)-t}}} \\
& \in \Gamma^{\infty}\left(\wedge^{t} T V^{\alpha \beta}\right) \otimes \Gamma^{\infty}\left(\wedge^{k-l(\beta)-t} T N^{\alpha}\right) .
\end{aligned}
$$

The component of $L\left(T\left(\xi_{(\alpha)}\right) \circ T\left(\eta_{(\beta)}\right)\right)$ in

$$
\Gamma^{\infty}\left(\wedge^{k+l-l(g)-1} T V^{g} \otimes \wedge^{l(g)}\left(N^{\alpha} \oplus N^{\beta}\right)\right)^{C(g)}
$$

is computed to be

$$
\begin{aligned}
& \sum_{\substack{g=\lambda \mu \\
\lambda \in(\alpha), \mu \in(\beta) \\
l(g)=l(\lambda)+l(\mu)}} \sum_{i_{1}, \ldots, i_{k-l(\lambda)}, j_{1}, \ldots, j_{l-l(\mu)}}(-1)^{(z-1)(l-1)+(k-z) l+(l-l(\mu)) l(\lambda)} X_{\lambda}^{i_{1} \cdots \hat{i}_{z} \cdots i_{k-l(\lambda)}} \\
& \frac{\partial}{\partial x^{i_{z}}} Y_{\mu}^{j_{1} \cdots j_{l-l(\mu)}} \frac{\partial}{\partial x^{i_{1}}} \wedge \cdots \frac{\widehat{\partial}}{\partial x^{i_{z}}} \cdots \wedge \frac{\partial}{\partial x^{i_{k-l(\lambda)}}} \wedge \frac{\partial}{\partial x^{j_{1}}} \wedge \cdots \wedge \frac{\partial}{\partial x^{j_{l-l(\mu)}}} \otimes \Lambda_{\lambda} \wedge \Lambda_{\mu} \\
& +\sum_{i_{1}, \ldots, i_{k-l(\lambda)}, j_{1}, \ldots, j_{l-l(\mu)-1}, q_{z}}(-1)^{(k-l(\lambda))(l-1)+(k-l(\mu))(l(\lambda)-1)} X_{\lambda}^{i_{1} \cdots i_{k-l(\lambda)}} \\
& \frac{\partial}{\partial x^{q_{z}}} Y_{\mu}^{j_{1}, \ldots, j_{l-l(\mu)-1}, q_{z}} \frac{\partial}{\partial x^{i_{1}}} \wedge \cdots \wedge \frac{\partial}{\partial x^{i_{k-l(\lambda)}}} \wedge \frac{\partial}{\partial x^{j_{1}}} \wedge \cdots \wedge \frac{\partial}{\partial x^{j_{l-l(\mu)-1}}} \otimes \Lambda_{\lambda} \wedge \Lambda_{\mu} .
\end{aligned}
$$

Proof. The first statement is a corollary of Lemma 4.8 It remains to show the second statement.

As $G$ is abelian, we can simultaneously diagonalize the $\alpha, \beta$ action on $V$. Using the fact that $V^{\alpha}+V^{\beta}=V$, we can write $V=V^{\alpha \beta} \oplus N^{\alpha} \oplus N^{\beta}$ such that $V^{\alpha}=$ $V^{\alpha \beta} \oplus N^{\beta}$ and $V^{\beta}=V^{\alpha \beta} \oplus N^{\alpha}$.

By Lemma 4.3, we conclude that $X_{\alpha}$ must be from $\Gamma^{\infty}\left(\wedge^{k-l(\alpha)} T V^{\alpha \beta}\right)$ to have a nontrivial contribution in $L\left(\left[T\left(\xi_{(\alpha)}\right), T\left(\eta_{(\beta)}\right)\right]\right)$. 
Similarly, by Lemma 4.9, we conclude that to have a nontrivial contribution in $L\left(\left[T\left(_{(\alpha)}\right), T\left(\eta_{(\beta)}\right)\right]\right)$, the direction $Y_{\beta}$ has to be from one of the following spaces:

(i) $Y_{\beta} \in \Gamma^{\infty}\left(\wedge^{l-l(\beta)} T V^{\alpha \beta}\right), \quad$ (ii) $Y_{\beta} \in \Gamma^{\infty}\left(\wedge^{l-l(\beta)-1} T V^{\alpha \beta}\right) \otimes \Gamma^{\infty}\left(T N^{\alpha}\right)$.

We apply Theorem 4.4 to compute the pre-Lie product. Because both $V^{\alpha \beta}$ and $N^{\alpha}$ are subspaces of $V^{\beta}$, which is the fixed point set of $\beta$, we can drop the $\beta$ twist of the pre-Lie product. Therefore, we are left with the standard pre-Lie product.

When $Y_{\beta} \in \Gamma^{\infty}\left(\wedge^{l-l(\beta)} T V^{\alpha \beta}\right)$, we have $L\left(\left[T\left(\xi_{\alpha}\right), T\left(\eta_{\beta}\right)\right]\right)=$

$$
\begin{gathered}
\sum_{i_{1}, \ldots, i_{k-l(\alpha)}, j_{1}, \ldots, j_{l-l(\beta)}}(-1)^{(z-1)(l-1)+(k-z) l+(l-l(\beta)) l(\alpha)} X_{\alpha}^{i_{1} \cdots \hat{i_{z}} \cdots i_{k-l(\alpha)}} \\
\frac{\partial}{\partial x^{i_{z}}} Y_{\beta}^{j_{1} \cdots j_{l-l(\beta)}} \frac{\partial}{\partial x^{i_{1}}} \wedge \cdots \frac{\widehat{\partial}}{\partial x^{i_{z}}} \cdots \wedge \frac{\partial}{\partial x^{i_{k-l(\alpha)}}} \wedge \frac{\partial}{\partial x^{j_{1}}} \wedge \cdots \wedge \frac{\partial}{\partial x^{j_{l-l(\beta)}}} .
\end{gathered}
$$

When $Y_{\beta} \in \Gamma^{\infty}\left(\wedge^{l-l(\beta)-1} T V^{\alpha \beta}\right) \otimes \Gamma^{\infty}\left(T N^{\alpha}\right)$, we compute $L\left(\left[T\left(\xi_{\alpha}\right), T\left(\eta_{\beta}\right)\right]\right)=$

$$
\begin{gathered}
\sum_{i_{1}, \ldots, i_{k-l(\alpha)}, j_{1}, \ldots, j_{l-l(\beta)-1}, q_{z}}(-1)^{(k-l(\alpha))(l-1)+(k-l(\beta))(l(\alpha)-1)} X_{\alpha}^{i_{1} \cdots i_{k-l(\alpha)}} \\
\frac{\partial}{\partial q_{z}} Y_{\beta}^{j_{1}, \ldots, j_{l-l(\beta)-1}, q_{z}} \frac{\partial}{\partial x^{i_{1}}} \wedge \cdots \wedge \frac{\partial}{\partial x^{i_{k-l(\alpha)}}} \wedge \frac{\partial}{\partial x^{j_{1}}} \wedge \cdots \wedge \frac{\partial}{\partial x^{j_{l-l(\beta)-1}}} .
\end{gathered}
$$

Corollary 4.11. Let $G$ be an abelian group acting a manifold $M$. The $(g)$ component of the twisted Schouten-Nijenhuis bracket is

$$
L([T(\xi), T(\eta)])=p r^{g}\left(\left.\sum_{\substack{g=\lambda \mu \\ \lambda \in(\alpha), \mu \in(\beta) \\ l(g)=l(\lambda)+l(\mu)}}\left[\tilde{\xi}_{\lambda}, \tilde{\eta}_{\mu}\right]\right|_{\left.M^{\lambda \mu}\right) .}\right.
$$

\section{Noncommutative Poisson structure \\ AND SYMPLECTIC REFLECTION ALGEBRAS}

In this section, we want to find geometric expressions of all possible noncommutative Poisson structures on $C^{\infty}(M) \rtimes G$.

5.1. Noncommutative Poisson structure. We assume that the manifold $M$ has a $G$-invariant symplectic structure. Because the group $G$ is finite, there always exists a $G$-invariant compatible almost complex structure $J$ on $M$. Accordingly, for any $g$ in $G$, the fixed point manifold $M^{g}$ is again a symplectic manifold with a compatible almost complex structure [9]. Therefore, all the fixed point manifolds $M^{g}, g \in G$ are of even dimension. Furthermore, the restriction of the $l(g)$-th wedge power of the corresponding Poisson structure defines a global section on $\wedge^{l(g)} N^{g}$. Therefore, we can choose $\Lambda_{g}$ to be constant for any $g \in G$.

Since all the fixed point manifolds have at least codimension equal to 2 , there is no contribution of $\Gamma^{\infty}\left(\bigoplus_{g ; l(g)=1} T M^{g} \otimes N^{g}\right)^{G}$ in the Hochschild cohomology of $C^{\infty}(M) \rtimes G$. 
For a noncommutative Poisson structure on $C^{\infty}(M) \rtimes G$, we need to consider degree 2 Hochschild cohomology classes. Since there is no contribution from $g$-fixed point submanifolds with $l(g)=1$ (Equation (2) ), we have the following description of the degree 2 Hochschild cohomology of $C^{\infty}(M) \rtimes G$ :

$$
H H^{2}\left(C^{\infty}(M) \rtimes G, C^{\infty}(M) \rtimes G\right)=\Gamma^{\infty}\left(\wedge^{2} T M\right)^{G} \oplus \Gamma^{\infty}\left(\bigoplus_{g \in G, l(g)=2} \wedge^{2} N^{g}\right)^{G}
$$

where the $G$-action on the second component is by conjugation. Inspired by the above equation, we define the set $S$ of elements $g \in G$ such that the fixed point subspace of $g$ is of codimension 2. It is easy to see that $S$ is closed under the conjugate action of $G$.

With the above discussion, we are ready to state the following geometric description of noncommutative Poisson structures on $C^{\infty}(M) \rtimes G$.

We assume that the manifold $M$ has a $G$-invariant symplectic structure. As $G$ is finite, there always exists a $G$-invariant compatible almost complex structure $J$ on $M$. Therefore, for any $g$ in $G$, the fixed point manifold $M^{g}$ is a symplectic submanifold with a compatible almost complex structure [9] and accordingly is of even dimension. Furthermore, we have that the restriction of the $l(g)$-th wedge power of the corresponding Poisson structure defines a global section on $\wedge^{l(g)} N^{g}$, which is $G$-invariant.

Theorem 5.1. Assume that $M$ is a symplectic manifold 5 with a $G$ symplectic action. Let $\pi$ be an element in $\Gamma^{\infty}\left(\wedge^{2} T M\right)^{G}$, and $\sum_{g \in S} \Lambda_{g}$ be an element in $\left(\bigoplus_{g \in S} \wedge^{2} N^{g}\right)^{G}$. Then $\pi+\sum_{g} \Lambda_{g}$ is a noncommutative Poisson structure on $C^{\infty}(M) \rtimes G$ if and only if

(1) on $M,[\pi, \pi]=0$;

(2) for any $g \in S, \operatorname{pr}^{g}\left(\left.\left[\pi, \widetilde{\Lambda}_{g}\right]\right|_{M^{g}}\right)=0$.

In the above, we have assumed that there is no group element of $G$ except the identity that is a stabilizer of an open subset of $M$. In this case, we call the $G$-action reduced.

Proof. We compute $L\left(\left[T\left(\pi+\sum_{g \in S} \Lambda_{g}\right), T\left(\pi+\sum_{g \in S} \Lambda_{g}\right)\right]\right)$. It decomposes into the sum of four terms:

$$
\begin{array}{cc}
\text { i) } L([T(\pi), T(\pi)]), & \text { ii) } \sum_{\alpha \in S} L\left(\left[T(\pi), T\left(\Lambda_{\alpha}\right)\right]\right), \\
\text { iii) } \sum_{\alpha \in S} L\left(\left[T\left(\Lambda_{\alpha}\right), T(\pi)\right]\right), & \text { iv) } \sum_{\alpha, \beta \in S} L\left(\left[T\left(\Lambda_{\alpha}\right), T\left(\Lambda_{\beta}\right)\right]\right) .
\end{array}
$$

We compute the above $i)$-iv) terms separately.

(i) $L([T(\pi), T(\pi)])$. On the identity component, the Gerstenhaber bracket corresponds to the standard Schouten-Nijenhuis bracket. Therefore, we have

$$
L([T(\pi), T(\pi)])=[\pi, \pi]
$$

which is again on the identity component.

\footnotetext{
$M$.

${ }^{5}$ From the proof, we see that all we really need is a $G$-invariant almost complex structure on
} 
(ii) $\sum_{\alpha \in S} L\left(\left[T(\pi), T\left(\Lambda_{\alpha}\right)\right]\right)$. The identity component contributes $\pi$ and the $\alpha$ component contributes $\Lambda_{\alpha}$. We use Example 4.6 to compute these terms,

$$
\sum_{\alpha \in S} L\left(\left[T(\pi), T\left(\Lambda_{\alpha}\right)\right]\right)=\sum_{\alpha \in S} p^{\alpha}\left(\left.\left[\pi, \widetilde{\Lambda}_{\alpha}\right]\right|_{M^{\alpha}}\right)
$$

where $\left.\left[\pi, \widetilde{\Lambda}_{\alpha}\right]\right|_{M^{\alpha}}$ is on the $\alpha$ component.

(iii) $\left.\sum_{\alpha \in S} L\left(\left[T\left(\Lambda_{\alpha}\right), T(\pi)\right]\right)\right)$. This case is similar to the previous case. We apply Example 4.6 to compute the terms

$$
\sum_{\alpha \in S} L\left(\left[T\left(\Lambda_{\alpha}\right), T(\pi)\right]\right)=\sum_{\alpha \in S} p r^{\alpha}\left(\left[\widetilde{\Lambda}_{\alpha}, \pi\right]_{M^{\alpha}}\right),
$$

where $\left[\widetilde{\Lambda}_{\alpha}, \pi\right]_{M^{\alpha}}$ is on the $\alpha$ component.

(iv) $\sum_{\alpha, \beta \in S} L\left(\left[T\left(\Lambda_{\alpha}\right), T\left(\Lambda_{\beta}\right)\right]\right)$. We need to use the assumption that $M$ is a symplectic manifold. Accordingly, $M^{\alpha}$ is a symplectic submanifold of codimension 2. Furthermore, if we fix a $G$-invariant compatible almost complex structure on $M, M^{\alpha}$ is an almost complex submanifold. Hence, for each $x \in M^{\alpha} \cap M^{\beta}$ with $\alpha, \beta \in S, T_{x} M^{\alpha}$ and $T_{x} M^{\beta}$ are almost complex subspaces of $T_{x} M$. The sum $T_{x} M^{\alpha}+T_{x} M^{\beta}$ is again an almost complex subspace of $T_{x} M$ and satisfies one of the following properties:

(a) $T_{x} M^{\alpha}+T_{x} M^{\beta}=T_{x} M$,

(b) $T_{x} M^{\alpha}=T_{x} M^{\beta}=T_{x} M^{\alpha \beta}$,

(c) $T_{x} M^{\alpha}=T_{x} M^{\beta}$ with $T_{x} M^{\alpha \beta}=T_{x} M$.

We discuss these three cases separately.

(a) If $T_{x} M^{\alpha}+T_{x} M^{\beta}=T_{x} M$, then by Lemma 4.5. $T_{x} M^{\alpha \beta}=T_{x} M^{\alpha} \cap T_{x} M^{\beta}$. Therefore, $M^{\alpha \beta}$ is a codimension 4 submanifold near $x$. We notice that $\left[T\left(\Lambda_{\alpha}\right), T\left(\Lambda_{\beta}\right)\right]$ is a 3 -cocycle. By Equation (2) with $\bullet=3$, we see that any contribution to the degree 3 Hochschild cohomology of $C^{\infty}(M) \rtimes G$ from a $g$-fixed point submanifold with $l(g)=4$ is trivial. Hence, the $\alpha \beta$ component of $\sum_{\alpha, \beta \in S} L\left(\left[T\left(\Lambda_{\alpha}\right), T\left(\Lambda_{\beta}\right)\right]\right)(x)$ vanishes.

(b) If $T_{x} M^{\alpha}=T_{x} M^{\beta}=T_{x} M^{\alpha \beta}$, then $M^{\alpha}=M^{\beta}=M^{\alpha \beta}=M^{\alpha} \cap M^{\beta}$ near $x$. Therefore, we apply Lemma 4.1 to compute $L\left(T\left(\Lambda_{\alpha}\right) \circ T\left(\Lambda_{\beta}\right)\right)$ and $L\left(T\left(\Lambda_{\beta}\right) \circ T\left(\Lambda_{\alpha}\right)\right)$ by the twisted pre-Lie product (Definition 4.2). We observe that the normal bundles $N^{\alpha}$ and $N^{\beta}$ are both symplectic orthogonal to $M^{\alpha}=M^{\beta}$. Therefore, $N^{\alpha}$ is the same as $N^{\beta}$. In Definition 4.2, since the $\beta$-action preserves $N^{\beta}=N^{\alpha}$, to have a nontrivial outcome we need all 3 terms $\left(x_{s}-x\right)^{i_{s}}$ for $s=1, \ldots, 3$ to be along the $N^{\alpha}=N^{\beta}$ direction.

This is because $\Lambda_{\alpha}$ and $\Lambda_{\beta}$ both contains 2 derivations along $N^{\alpha}=$ $N^{\beta}$. On the other hand, $N^{\alpha}=N^{\beta}$ is only of 2 dimensions. Any wedge product of 3 vectors along $N^{\alpha}$ vanishes. Therefore, the $\alpha \beta$ component of $\sum_{\alpha, \beta \in S} L\left(\left[T\left(\Lambda_{\alpha}\right), T\left(\Lambda_{\beta}\right)\right]\right)(x)$ vanishes.

(c) If $T_{x} M^{\alpha}=T_{x} M^{\beta}$ but $T_{x} M^{\alpha \beta}=T_{x} M$, then this shows that $\alpha \beta$ acts on $T_{x} M$ trivially and therefore there is a neighborhood of $x$ which is totally fixed by $\alpha \beta$. Hence, by the assumption that the $G$-action on $M$ is reduced, we know $\alpha \beta=1$. (As $N^{\alpha}=N^{\beta}$ is of 2 dimensions, the action of the centralizer subgroups $C(\alpha)$ and $C(\beta)$ on $N^{\alpha}$, which are subgroups of $U(1)$, can be diagonalized simultaneously. We choose the eigenfunctions of this action to be the coordinate functions. See Lemma 3.2.) We notice that 
$T\left(\Lambda_{\alpha}\right)$ and $T\left(\Lambda_{\beta}\right)$ both contains 2 derivations along the $N^{\alpha}=N^{\beta}$ direction. It is not difficult to check that $L\left(\left[T\left(\Lambda_{\alpha}\right), T\left(\Lambda_{\beta}\right)\right]\right)$ contains the wedge product of 3 vector fields along the $N^{\alpha}=N^{\beta}$ direction. Therefore, the identity component of $L\left(\left[T\left(\Lambda_{\alpha}\right), T\left(\Lambda_{\beta}\right)\right]\right)(x)$ vanishes for the same reason as in part (b).

In summary, we have that

$$
\begin{aligned}
& L\left(\left[T\left(\pi+\sum_{g \in S} \Lambda_{g}\right), T\left(\pi+\sum_{g} \Lambda_{g}\right)\right]\right) \\
= & {[\pi, \pi]+\sum_{\alpha \in S} \operatorname{pr}^{\alpha}\left(\left.\left[\pi, \tilde{\Lambda}_{\alpha}\right]\right|_{M^{\alpha}}\right)+\sum_{\alpha \in S} \operatorname{pr}^{\alpha}\left(\left.\left[\tilde{\Lambda}_{\alpha}, \pi\right]\right|_{M^{\alpha}}\right) } \\
= & {[\pi, \pi]+2 \sum_{\alpha \in S} \operatorname{pr}^{\alpha}\left(\left.\left[\pi, \tilde{\Lambda}_{\alpha}\right]\right|_{M^{\alpha}}\right) . }
\end{aligned}
$$

Therefore, if $\pi+\sum_{g \in S} \Lambda_{g}$ is a noncommutative Poisson structure, then $[\pi, \pi]=0$ and $p r^{\alpha}\left(\left.\left[\pi, \tilde{\Lambda}_{\alpha}\right]\right|_{M^{\alpha}}\right)=0$ for any $\alpha \in S$.

Corollary 5.2. Let $V$ be a real symplectic vector space with a $G$-invariant linear symplectic form $\omega$. Assume that the $G$-action on $V$ is reduced as in Theorem 5.1 . Let $\pi$ be the corresponding Poisson structure of $\omega$. Then $\kappa=\pi+\sum_{\alpha \in S} \Lambda_{\alpha}$ is a noncommutative Poisson structure on $\operatorname{Poly}(V) \rtimes G$ if and only if $\Lambda_{\alpha}$ is constant on $V^{\alpha}$.

Proof. Under the assumption of the corollary, the restriction of the symplectic form $\omega$ to $N^{\alpha}$ for $\alpha \in S$ is a symplectic two-form. We denote the corresponding Poisson structure on $N^{\alpha}$ by $\pi_{\alpha}$. Accordingly, we can write $\Lambda_{\alpha}=f_{\alpha} \pi_{\alpha}$, where $f_{\alpha}$ is a polynomial function on $V^{\alpha}$.

By Theorem 5.1, $\pi+\sum_{\alpha \in S} f_{\alpha} \pi_{\alpha}$ is a noncommutative Poisson structure if and only if

(1) $[\pi, \pi]=0$,

(2) $\left.\left[\pi, f_{\alpha}\right]\right|_{V^{\alpha}}=0$.

Equation (1) is automatically satisfied because $\pi$ is Poisson. Because $\left[\pi, \pi_{\alpha}\right]=0$, Equation (2) is reduced to

$$
\operatorname{pr}^{\alpha}\left(\left.\left[\pi, f_{\alpha} \pi_{\alpha}\right]\right|_{V^{\alpha}}\right)=\operatorname{pr}^{\alpha}\left(\left.\left[\pi, f_{\alpha}\right] \wedge \pi_{\alpha}\right|_{V^{\alpha}}\right)=\left.\left[\pi, f_{\alpha}\right]\right|_{V^{\alpha}} \wedge \pi_{\alpha}=0 .
$$

In the second equality, we have used the fact that as $f_{\alpha}$ is a function on $V^{\alpha}$, the bracket $\left[\pi, f_{\alpha}\right]$ is a vector field along $V^{\alpha}$. Therefore, $\pi+\sum_{\alpha \in S} f_{\alpha} \pi_{\alpha}$ is a noncommutative Poisson structure if and only if $\left.\left[\pi, f_{\alpha}\right]\right|_{V^{\alpha}}=\left[\pi, f_{\alpha}\right]=0$, for all $\alpha \in S$. Because $\omega$ is symplectic, $\left[\pi, f_{\alpha}\right]=0$ forces $f_{\alpha}$ to be a constant. Therefore, $\Lambda_{\alpha}$ is also a constant on $V^{\alpha}$ for all $\alpha \in S$.

5.2. Remarks on deformation quantizations. It is known that the set of noncommutative Poisson structures on an algebra $A$ is in one-to-one correspondence to the set of infinitesimal deformations of $A$. It is natural to ask whether one can integrate the infinitesimal deformation associated to a noncommutative Poisson structure to a real one. This question relates to the idea of deformation quantization in mathematical physics. In [16], the second author introduced a notion of deformation quantization of a noncommutative Poisson structure, which we now recall. 
Definition 5.3. A deformation quantization of a noncommutative Poisson structure $\Pi$ on an associative algebra $A$ is an associative product $\star_{\hbar}$ on $A[[\hbar]]$, such that $f \star_{\hbar} g=\sum_{i} \hbar^{i} C_{i}(f, g)$ for all $f, g \in A$ satisfying the following conditions:

(1) $C_{0}(f, g)=f g$;

(2) the Hochschild cohomology class $\left[C_{1}\right]$ is equal to $\Pi$.

It is natural to ask whether all the noncommutative Poisson structures defined in Theorem 5.1 can be deformation quantized. One special case has already been studied extensively, namely, when the noncommutative Poisson structure comes from a $G$-invariant Poisson structure on $M$ (i.e., when there are no components supported on fixed point submanifolds of codimension 2). The deformation quantizations of these types of noncommutative Poisson structures on $C^{\infty}(M) \rtimes G$ were studied in [16, 17, [5], 13, etc. Another well-studied and well-known special case is the following proposition, which is essentially due to Etingof and Ginzburg [7, Theorem 1.3].

Proposition 5.4. The noncommutative Poisson structure on a symplectic vector space obtained in Corollary 5.2 can be deformation quantized.

Proof. In the following, we work with the field $\mathbb{C}$, because we will use the construction of the symplectic reflection algebras in $[7]$ and Theorem 1.3 therein. Everything extends to the field $\mathbb{R}$, because Theorem 1.3 in 7 still holds in the real case. (The real group algebra of a finite group is semisimple.)

In [7, a symplectic reflection algebra $H_{t, c}$ is introduced as

$$
T V \rtimes G /\left\langle x \otimes y-y \otimes x-\kappa(x, y) \in T^{2} V \oplus \mathbb{C} G\right\rangle_{x, y \in V},
$$

where $(V, \omega)$ is a finite dimensional complex symplectic vector space over $\mathbb{C}$, and $T V$ is its tensor algebra, and $T^{2} V$ is $V \otimes V$, and $\kappa$ is defined to be

$$
\kappa(x, y)=t \pi(x, y)+\sum_{\alpha \in S} c_{\alpha} \pi_{\alpha} U_{\alpha}
$$

a $G$-invariant section of $\wedge^{2} V+\bigoplus_{\alpha \in S} \wedge^{2} N^{\alpha}$.

We assign $V$ degree 1 , and $\mathbb{C} G$ degree 0 . This defines an increasing filtration $F_{\bullet}$ on $H_{t, c}$. It was proved by Etingof and Ginzburg in [7, Theorem 1.3] that $H_{t, c}$ satisfies the Poincaré-Birkhoff-Witt property, i.e. that tautological embedding $V \hookrightarrow \operatorname{gr}\left(H_{t, c}\right)$ extends to an isomorphism $Q: \operatorname{Poly}(V) \rtimes G \rightarrow \operatorname{gr}\left(H_{t, c}\right)$ of vector spaces. We define $g r_{i}$ to be the projection from $g r\left(H_{t, c}\right)$ to its $i$ th degree component.

We define a formal deformation quantization of $\operatorname{Poly}(V) \rtimes G$ as follows. For $f U_{\alpha}, g U_{\beta} \in \operatorname{Poly}(V) \rtimes G$,

$$
f U_{\alpha} \star g U_{\beta}=\sum_{i, j, k=0}^{\infty} \hbar^{i+j-k} Q^{-1}\left(g r_{k}\left(g r_{i}\left(Q\left(f U_{\alpha}\right)\right) g r_{j}\left(Q\left(g U_{\beta}\right)\right)\right)\right) .
$$

In particular, $C_{i}\left(f U_{\alpha}, g U_{\beta}\right)$ is defined to be

$$
C_{i}\left(f U_{\alpha}, g U_{\beta}\right)=\sum_{p+q-r=i} Q^{-1}\left(g r_{r}\left(g r_{p}\left(Q\left(f U_{\alpha}\right)\right) g r_{q}\left(Q\left(g U_{\beta}\right)\right)\right)\right) .
$$

Because $Q\left(f U_{\alpha}\right)$ and $Q\left(g U_{\beta}\right)$ are of finite degrees, $p, q$ in the summation are both finite. Therefore, the sum in the definition of $C_{i}$ is finite and $C_{i}$ is well defined. 
We check that $\star$ is associative. $\left(f U_{\alpha} \star g U_{\beta}\right) \star h U_{g}$

$$
\begin{aligned}
= & \sum_{i, j, k=0}^{\infty} \hbar^{i+j-k} Q^{-1}\left(g r_{k}\left(g r_{i}\left(Q\left(f U_{\alpha}\right)\right) g r_{j}\left(Q\left(g U_{\beta}\right)\right)\right)\right) \star h U_{g} \\
= & \sum_{i, j, k, p, q, r=0}^{\infty} \hbar^{i+j-k} \hbar^{p+q-r} Q^{-1}\left(g r _ { r } \left(g r _ { p } \left(Q\left(Q^{-1}\left(g r_{k}\left(g r_{i}\left(Q\left(f U_{\alpha}\right) g r_{j}\left(Q\left(g U_{\beta}\right)\right)\right)\right)\right)\right)\right.\right.\right. \\
= & \sum_{i, j, k=0}^{\infty} \sum_{p, q, r=0}^{\infty} \hbar^{i+j+p+q-k-r} Q^{-1}\left(g r_{r}\left(g r_{p}\left(g r_{k}\left(g r_{i}\left(Q\left(f U_{\alpha}\right)\right) g r_{j}\left(Q\left(g U_{\beta}\right)\right)\right)\right) g r_{q}\left(Q\left(h U_{g}\right)\right)\right)\right) \\
= & \sum_{i, j, k=p, q, r}^{\infty} \hbar^{i+j+q-r} Q^{-1}\left(g r_{r}\left(g r_{k}\left(g r_{i}\left(Q\left(f U_{\alpha}\right)\right) g r_{j}\left(Q\left(g U_{\beta}\right)\right)\right) g r_{q}\left(Q\left(h U_{g}\right)\right)\right)\right) \\
= & \sum_{i, j, q=0}^{\infty} \hbar^{i+j+q} \sum_{r=0}^{\infty} \hbar^{i+j+q-r} Q^{-1}\left(g r_{r}\left(\sum_{k=0}^{\infty} g r_{k}\left(g r_{i}\left(Q\left(f U_{\alpha}\right)\right) g r_{j}\left(Q\left(g U_{\beta}\right)\right)\right) g r_{q}\left(Q\left(h U_{g}\right)\right)\right)\right) \\
= & \sum_{i, j, k, r=0}^{\infty} \hbar^{i+j+q-r} Q^{-1}\left(g r_{r}\left(g r_{i}\left(Q\left(f U_{\alpha}\right)\right) g r_{j}\left(Q\left(g U_{\beta}\right)\right) g r_{q}\left(Q\left(h U_{g}\right)\right)\right)\right),
\end{aligned}
$$

which by a similar computation is equal to

$$
f U_{\alpha} \star\left(g U_{\beta} \star h U_{g}\right) .
$$

We look at $C_{1}\left(f U_{\alpha}, g U_{\beta}\right)=\sum_{i+j-k=1} Q^{-1}\left(g r_{k}\left(g r_{i}\left(Q\left(f U_{\alpha}\right)\right) g r_{j}\left(Q\left(g U_{\beta}\right)\right)\right)\right)$. To check that $C_{1}$ is cohomologous to $\kappa$, we compute $L\left(C_{1}\right)$ as follows using the definition of $L$ :

$$
\begin{aligned}
& =L_{3}\left(\sum_{i_{1}, i_{2}} C_{1}\left(x_{1}^{i_{1}}-x^{i_{1}}, x_{2}^{i_{2}}-x^{i_{2}}\right) \frac{\partial}{\partial x^{i_{1}}} \wedge \frac{\partial}{\partial x^{i_{2}}}\right) \\
& =L_{3}\left(\sum_{i_{1}, i_{2}} g r_{0}\left(\left(x_{1}-x\right)^{i_{1}}\left(x_{2}-x\right)^{i_{2}}\right) \frac{\partial}{\partial x^{i_{1}}} \wedge \frac{\partial}{\partial x^{i_{2}}}\right) \\
& =L_{3}\left(\sum_{i_{2}<i_{1}}\left(x^{i_{1}} x^{i_{2}}-x^{i_{2}} x^{i_{1}}\right) \frac{\partial}{\partial x^{i_{1}}} \wedge \frac{\partial}{\partial x^{i_{2}}}\right) \\
& =\sum_{i_{2}<i_{1}}\left(\omega\left(x^{i_{1}}, x^{i_{2}}\right)+\sum_{\alpha \in S} c_{\alpha} \omega_{\alpha}\left(x^{i_{1}}, x^{i_{2}}\right) U_{\alpha}\right) \frac{\partial}{\partial x^{i_{1}}} \wedge \frac{\partial}{\partial x^{i_{2}}} \\
& =\frac{1}{2} \sum_{i_{1}, i_{2}}\left(\omega\left(x^{i_{1}}, x^{i_{2}}\right)+\sum_{\alpha \in S} c_{\alpha} \omega_{\alpha}\left(x^{i_{1}}, x^{i_{2}}\right) U_{\alpha}\right) \frac{\partial}{\partial x^{i_{1}}} \wedge \frac{\partial}{\partial x^{i_{2}}} .
\end{aligned}
$$

In the third equality of the above equation, we have used the definition of $C_{1}$ and the product structure in $H_{t, c}$. When $i_{1}<i_{2}$, the term $x^{i_{1}} x^{i_{2}}$ has no degree 0 term. When $i_{1}>i_{2}$, the degree 0 term of $x^{i_{1}} x^{i_{2}}$ is $x^{i_{1}} x^{i_{2}}-x^{i_{2}} x^{i_{1}}$.

In conclusion, $\star$ is a deformation quantization of $A \rtimes G$ with the noncommutative Poisson structure equal to $\frac{1}{2} \kappa$.

Remark 5.5. The generalization of the deformation quantization defined in Proposition 5.4 to affine varieties was studied by Etingof [6.

It is natural to ask whether the deformation quantization constructed in Theorem 5.4 is unique up to isomorphism. From Poisson geometry, we know that 
the isomorphism classes of a deformation quantization of a Poisson structure are determined by its second Poisson cohomology, introduced in [18].

We briefly recall the definition of Poisson cohomology here. Let $\pi$ be a noncommutative Poisson structure on an $A$. As $[\pi, \pi]_{G}=0$ in $H H^{\bullet}(A, A)$, the operator $d^{\pi}: H H^{\bullet}(A, A) \rightarrow H H^{\bullet+1}(A, A)$, defined by

$$
d^{\pi}(\phi)=[\pi, \phi], \quad \phi \in H^{\bullet}(A, A),
$$

satisfies $d^{\pi} \circ d^{\pi}=0$. The cohomology of $H H^{\bullet}(A, A)$ with respect to $d^{\pi}$ is called the Poisson cohomology of $\pi$.

We denote the Poisson cohomology group of $\operatorname{Poly}(V) \rtimes G$ associated to $\kappa$ by $H_{\kappa}^{\bullet}(\operatorname{Poly}(V) \rtimes G)$. In the following we compute $H_{\kappa}^{2}(\operatorname{Poly}(V) \rtimes G)$.

Proposition 5.6. Let $(V, \omega)$ be a real symplectic vector space with a finite group $G$ symplectic action. Let $\kappa$ be defined as in Corollary 5.2. Then the second Poisson cohomology of $\kappa$ is isomorphic to

$$
\left\{\sum_{g \in S} c_{g} \pi_{g} \in \Gamma^{\infty}\left(\bigoplus_{g \in S} \wedge^{2} N^{g}\right)^{G} \mid \text { for all } g \in S, c_{g} \text { is a constant on } V^{g}\right\} .
$$

Proof. By [9], the fixed point subspace $V^{g}$ of $g$ is always a symplectic vector space, which implies that $V^{g}$ is of even dimension. According to Equation (1D) and the fact that the codimensions of all $V^{\alpha}$ are even, the second Hochschild cohomology of $\operatorname{Poly}(V) \rtimes G$ is

$$
\Gamma^{\infty}\left(\wedge^{2} T V\right)^{G} \oplus\left(\sum_{g \in S} \wedge^{0} V^{g} \otimes \wedge^{2} N^{g}\right)^{G}
$$

Let $\Xi+\sum_{g \in S} f_{g} \pi_{g}$ be an element of $\Gamma^{\infty}\left(\wedge^{2} T V\right)^{G} \oplus\left(\sum_{g \in S} \wedge^{0} V^{g} \otimes \wedge^{2} N^{g}\right)^{G}$. We compute $L\left(\left[T\left(\Xi+\sum_{g \in S} f_{g} \pi_{g}\right), T(\kappa)\right]\right)$ as follows. The computation is similar to computations in the proof of Theorem [5.1. There are four terms, which we deal with separately:

$$
\begin{array}{ll}
\text { 1) } L([T(\Xi), T(\pi)]), & \text { 2) } L\left(\left[T(\Xi), T\left(\sum_{g \in S} c_{g} \pi_{g}\right)\right]\right), \\
\text { 3) } L\left(\left[T\left(\sum_{g \in S} f_{g} \pi_{g}\right), T(\pi)\right]\right), & \text { 4) } L\left(\left[T\left(\sum_{g \in S} f_{g} \pi_{g}\right), T\left(\sum_{g \in S} c_{g} \pi_{g}\right)\right]\right) .
\end{array}
$$

The following computation follows similar reasoning to that found in the proof of Theorem [5.1]

1) $L([T(\Xi), T(\pi)])=[\Xi, \pi]$

2) $L\left(\left[T(\Xi), T\left(\sum_{g \in S} c_{g} \pi_{g}\right)\right]\right)=\sum_{g \in S} \operatorname{pr}^{g}\left(\left.c_{g}\left[\Xi, \pi_{g}\right]\right|_{V^{g}}\right)$;

3) $L\left(\left[T\left(\sum_{g \in S} f_{g} \pi_{g}\right), T(\pi)\right]\right)=\sum_{g \in S} p r^{g}\left(\left.\left[f_{g} \pi_{g}, \pi\right]\right|_{V^{g}}\right)=\left.\sum_{g \in S} p r^{g}\left(\left[f_{g}, \pi\right] \wedge \pi_{g}\right)\right|_{V^{g}}$

4) $L\left(\left[T\left(\sum_{g \in S} f_{g} \pi_{g}\right), T\left(\sum_{g \in S} c_{g} \pi_{g}\right)\right]\right)=0$. 
According to the above computation, $\Xi+\sum_{g \in S} f_{g} \pi_{g}$ is $\kappa$ closed if and only if it satisfies the following equations:

1) $[\Xi, \pi]=0$,

2) $\operatorname{pr}^{g}\left(\left.\left\{c_{g}\left[\Xi, \pi_{g}\right]+\left[f_{g}, \pi\right] \wedge \pi_{g}\right\}\right|_{V^{g}}\right)=0$, for all $g \in G$.

We denote the space of solutions to the above equations by $Z_{\kappa}^{2}$.

Next we compute the Poisson coboundary in $\left(\Gamma^{\infty}\left(\wedge^{2} T V\right) \bigoplus_{\alpha \in S} \wedge^{2} N^{g}\right)^{G}$.

According to Equation (11), the cohomology $H^{1}(\operatorname{Poly}(V) \rtimes G, \operatorname{Poly}(V) \rtimes G)$ is a $G$-invariant vector field on $V$, as $V^{g}$ for $g \neq i d$ has at least codimension 2 for all $g$. Let $X \in \Gamma^{\infty}(T V)^{G}$. We compute $L([T(\kappa), T(X)])$ using Example 4.6;

$$
L([T(\kappa), T(X)])=[\pi, X]+\sum_{g \in s} p r^{g}\left(\left.c_{g}\left[\pi_{g}, X\right]\right|_{V^{g}}\right) .
$$

We denote the space of elements in $\Gamma^{\infty}\left(\wedge^{2} T V\right)^{G} \oplus\left(\sum_{g \in S} \wedge^{0} V^{g}\right)^{G}$ of the above form by $B_{\kappa}^{2}$.

We want to find the quotient $Z_{\kappa}^{2} / B_{\kappa}^{2}$. Given $\Xi \in \Gamma^{\infty}\left(\wedge^{2} T V\right)^{G}$ with $[\Xi, \pi]=0$, we use the fact that $\pi$ is from a symplectic form to find a $G$-invariant vector field $X \in \Gamma^{\infty}(T V)$ such that $[\pi, X]=\Xi$. Because $L([T(\kappa), T(X)])=: \Xi+\sum_{g} h_{g} \pi_{g}=$ $[\pi, X]+\sum_{g \in S} c_{g} p^{g}\left(\left.\left[\pi_{g}, X\right]\right|_{V^{g}}\right)$ is $\kappa$-closed we conclude that

$$
\Xi+\sum_{g \in S} h_{g} \pi_{g}-\left(\Xi+\sum_{g} f_{g} \pi_{g}\right)=\sum_{g \in S}\left(h_{g}-f_{g}\right) \pi_{g}
$$

is also $\kappa$-closed. Substituting this expression into the second equation of (8) with $\Xi=0$, we have that for all $g \in S$,

$$
\operatorname{pr}^{g}\left(\left.\left[\pi,\left(h_{g}-f_{g}\right)\right] \wedge \pi_{g}\right|_{V^{g}}\right)=\left.\left.\left[\pi,\left(h_{g}-f_{g}\right)\right]\right|_{V^{g}} \wedge \pi_{g}\right|_{V^{g}}=0 .
$$

In the above equality, we have used the fact that $h_{g}-f_{g}$ is supported on $V^{g}$ and therefore $\left[\pi, h_{g}-f_{g}\right]$ is an element in $T V^{g}$ also supported on $V^{g}$. Accordingly, we have

$$
\left.\left[\pi, h_{g}-f_{g}\right]\right|_{V^{g}}=0 \Leftrightarrow\left[\pi, h_{g}-f_{g}\right]=0 .
$$

Because $\pi$ is symplectic, this implies that $h_{g}-f_{g}$ has to be a constant. It is obvious that $X+\sum_{g \in S}\left(h_{g}+c_{g}\right) \pi_{g}$ is $\kappa$-closed.

We are left to show that nonzero elements of the form $\sum_{g \in S} a_{g} \pi_{g}$ are not coboundaries for any constant $a_{g}, g \in S$. If $X \in \Gamma^{\infty}(T V)^{G}$ such that $L(T(\kappa), T(X))$ $=\sum_{g \in S} a_{g} \pi_{g}$, then by similar computations to those above, we have that

$$
[\pi, X]=0, \quad p r^{g}\left(\left.c_{g}\left[\pi_{g}, X\right]\right|_{V^{g}}\right)=a_{g} \pi_{g}
$$

for any $g \in S$.

As $\pi$ is from a symplectic form, $[\pi, X]=0$ implies that there is a function $f$ such that $X=[\pi, f]$. As $g$ acts on $V$ preserving the symplectic form, we are allowed to write $\pi$ as a sum of $\pi_{g}$ and $\pi^{g}$, where $\pi_{g}$ is a bivector supported along $N^{g}$, and $\pi^{g}$ is a bivector supported along $V^{g}$. Therefore, we have

$$
\left[\pi_{g}, X\right]=\left[\pi_{g},[\pi, f]\right]=\left[\pi_{g},\left[\pi_{g}, f\right]\right]+\left[\pi_{g},\left[\pi^{g}, f\right]\right] .
$$

We easily compute that $\left[\pi_{g},\left[\pi_{g}, f\right]\right] \equiv 0$ and $\operatorname{pr}^{g}\left(\left[\pi_{g},\left[\pi^{g}, f\right]\right]\right) \equiv 0$ using the fact $N^{g}$ is of dimension 2. This shows that $\left[\pi_{g}, X\right]$ contains no component proportional to $\pi_{g}$. Accordingly, $\mathrm{pr}^{g}\left(c_{g}\left[\pi_{g}, X\right]\right)=0=a_{g} \pi_{g}$. 
In conclusion, we see that the quotient space $Z_{\kappa}^{2} / B_{\kappa}^{2}$ is

$$
\left\{\sum_{g \in S} c_{g} \pi_{g} \in \Gamma^{\infty}\left(\bigoplus_{g \in S} \wedge^{2} N^{g}\right)^{G} \mid \text { for all } g \in S, c_{g} \text { is a constant on } V^{g}\right\} \text {. }
$$

Remark 5.7. Proposition 5.6 shows that the dimension of all infinitesimal deformations of a noncommutative Poisson structure $\kappa$ is equal to the size of the set $S$. Furthermore, it is easy to check that all the infinitesimal deformations actually correspond to Poisson structures. This gives another explanation of Corollary [5.2.

Propositions 5.4 and 5.6 inspire a series of interesting questions. The cocycle $\kappa$ is a very special type of noncommutative Poisson structure on $A \rtimes G$ defined in Theorem 5.1. In [11, with Jean-Michel Oudom, we proved that, with a mild assumption, all linear Poisson structures on $\operatorname{Poly}(V) \rtimes G$ can be quantized. This inspires the question of whether all the noncommutative Poisson structures defined in Theorem 5.1 can be deformation quantized. If their deformation quantizations exist, how many are there? All these problems have a general version on $C^{\infty}(M) \rtimes$ $G$. It is closely related to the Conjecture 1 in $[5$ by Dolgushev and Etingof. We plan to address these questions in the near future.

5.3. Noncommutative quadratic Poisson structures. In this subsection, we provide some new examples of noncommutative Poisson structures other than those in Corollary 5.2. We can easily see that these Poisson structures are not symplectic at all, and they should be viewed as generalized quadratic Poisson structures.

We consider the space of $\mathbb{R}^{4}=\mathbb{C} \times \mathbb{C}$ with the following $\mathbb{Z}_{n} \times \mathbb{Z}_{m}$ action, where $\mathbb{Z}_{n}=\mathbb{Z} / n \mathbb{Z}$ and $\mathbb{Z}_{m}=\mathbb{Z} / m \mathbb{Z}$. Let $\left(z_{1}, z_{2}\right)$ be holomorphic coordinates on $\mathbb{C}^{2}$, and $(k, l) \in \mathbb{Z}_{n} \times \mathbb{Z}_{m}$. Define

$$
\begin{aligned}
(k, l):\left(z_{1}, z_{2}\right) & \longrightarrow\left(\exp \left(\frac{2 k \pi i}{n}\right) z_{1}, \exp \left(\frac{2 l \pi i}{m}\right) z_{2}\right) \\
\left(\bar{z}_{1}, \bar{z}_{2}\right) & \longrightarrow\left(\exp \left(-\frac{2 k \pi i}{n}\right) \bar{z}_{1}, \exp \left(-\frac{2 l \pi i}{m}\right) \bar{z}_{2}\right) .
\end{aligned}
$$

The fixed point subspace of $(k, l) \in \mathbb{Z}_{n} \times \mathbb{Z}_{m}$ can be described explicitly:

(1) if $k \neq 0, l \neq 0$, the fixed point set of $(k, l)$ consists of only one point, the origin;

(2) if $k=0, l \neq 0,(0, l)$ 's fixed point set is $\mathbb{C} \times\{0\} \subset \mathbb{C} \times \mathbb{C}$;

(3) if $k \neq 0, l=0,(k, 0)$ 's fixed point set is $\{0\} \times \mathbb{C} \subset \mathbb{C} \times \mathbb{C}$;

(4) if $k=l=0$, $(0,0)$ 's fixed point space is $\mathbb{C} \times \mathbb{C}$.

To look for noncommutative Poisson structures on $\operatorname{Poly}\left(\mathbb{R}^{4}\right) \rtimes\left(\mathbb{Z}_{n} \times \mathbb{Z}_{m}\right)$, we only need to consider the fixed point space of the identity, which is $\mathbb{C}^{2}$, and the codimension 2 fixed point subspace of the form $\{0\} \times \mathbb{C}$ of $(k, 0)$, and the fixed point subspace $\mathbb{C} \times\{0\}$ of $(0, l)$. We consider the following collection of bivector fields, where $\alpha, \beta, \lambda_{k}, \mu_{l}$ are real constants.

(1) On the fixed point subspace of $(0,0)$, we consider $\Pi_{0,0}^{\alpha}=i \alpha\left|z_{2}\right|^{2} \frac{\partial}{\partial z_{1}} \wedge \frac{\partial}{\partial \bar{z}_{1}}$. We notice that $\Pi_{0,0}$ is $\mathbb{Z}_{n} \times \mathbb{Z}_{m}$ invariant, and satisfies $\left[\Pi_{0,0}, \Pi_{0,0}\right]=0$.

(2) On the fixed point subspace of $(k, 0)$, we consider $\Pi_{k, 0}=i \lambda_{k}\left|z_{2}\right|^{2} \frac{\partial}{\partial z_{1}} \wedge \frac{\partial}{\partial \bar{z}_{1}}$, which is a smooth section of the determinant bundle of the normal bundle over $\{0\} \times \mathbb{C} \subset \mathbb{C} \times \mathbb{C}$. Again we notice that $\Pi_{k, 0}$ is $\mathbb{Z}_{n} \times \mathbb{Z}_{m}$ invariant, and $\left[\Pi_{0,0}, \Pi_{k, 0}\right]=0$. 
(3) On the fixed point subspace of $(0, l)$, we consider $\Pi_{0, l}=i \mu_{l}\left|z_{1}\right|^{2} \frac{\partial}{\partial z_{2}} \wedge \frac{\partial}{\partial \bar{z}_{2}}$, which is a smooth section of the determinant bundle of the normal bundle over $\mathbb{C} \times\{0\} \subset \mathbb{C} \times \mathbb{C}$. We notice that $\Pi_{0, l}$ is $\mathbb{Z}_{n} \times \mathbb{Z}_{m}$ invariant, but

$$
\begin{aligned}
{\left[\Pi_{0,0}, \Pi_{0, l}\right] } & =-\alpha \mu_{l}\left[\left|z_{2}\right|^{2}\left(z_{1} \frac{\partial}{\partial z_{1}}-\bar{z}_{1} \frac{\partial}{\partial \bar{z}_{1}}\right) \wedge \frac{\partial}{\partial z_{2}} \wedge \frac{\partial}{\partial \bar{z}_{2}}\right. \\
& \left.+\left|z_{1}\right|^{2}\left(z_{2} \frac{\partial}{\partial z_{2}}-\bar{z}_{2} \frac{\partial}{\partial \bar{z}_{2}}\right) \wedge \frac{\partial}{\partial z_{1}} \wedge \frac{\partial}{\partial \bar{z}_{1}}\right] \neq 0 .
\end{aligned}
$$

However, if we look at the restriction of $\left[\Pi_{0,0}, \Pi_{0, l}\right]$ to the fixed point subspace of $(0, l)$, which is $\mathbb{C} \times\{0\}$, it does vanish. We have that

$$
\operatorname{pr}^{(0, l)}\left(\left.\left[\Pi_{0,0}, \Pi_{0, l}\right]\right|_{\mathbb{C} \times\{0\}}\right)=0 .
$$

By Theorem [5.1, we conclude that the collection of $\left(\Pi_{0,0}^{\alpha}, \Pi_{k, 0}, \Pi_{0, l}\right)$ defines a family of noncommutative Poisson structures on $\operatorname{Poly}\left(\mathbb{R}^{4}\right) \rtimes\left(\mathbb{Z}_{n} \times \mathbb{Z}_{m}\right)$. One can also easily check that if we replace $\Pi_{0,0}^{\alpha}$ by $\Pi_{0,0}^{\beta}=i \beta\left|z_{1}\right|^{2} \frac{\partial}{\partial z_{2}} \wedge \frac{\partial}{\partial \bar{z}_{2}},\left(\Pi_{0,0}^{\beta}, \Pi_{k, 0}, \Pi_{0, l}\right)$ defines another family of noncommutative Poisson structures on $\operatorname{Poly}\left(\mathbb{R}^{4}\right) \rtimes\left(\mathbb{Z}_{n} \times\right.$ $\left.\mathbb{Z}_{m}\right)$.

There are various ways to generalize the above families of examples to higher dimensions. For example, one can consider actions of $\mathbb{Z}_{n} \times \mathbb{Z}_{m}$ which act on the first two components of $\mathbb{C}^{k}$ as above, but act trivially on the left $\mathbb{C}^{k-2}$ component. Then the above two families of noncommutative Poisson structures naturally extend to $\operatorname{Poly}\left(\mathbb{R}^{2 k}\right) \rtimes\left(\mathbb{Z}_{n} \times \mathbb{Z}_{m}\right)$. We will leave the more nontrivial generalizations to the future [1].

\section{ACKNOWLEDGMENTS}

We would like to thank Vassiliy Dolgushev, Benjamin Enriquez, Pavel Etingof, Victor Ginzburg, Jean-Michel Oudom, Hessel Posthuma, and Markus Pflaum for useful conversations. We would also like to particularly thank Ping $\mathrm{Xu}$ for hosting our visits to Penn State University in Spring 2005, where we started this project.

\section{REFERENCES}

1. Anno, R., Multiplicative structure on the Hochschild cohomology of crossed product algebras, preprint: math.QA/0511396.

2. Block, J. and Getzler, E.: Quantization of foliations, Proceedings of the XXth International Conference on Differential Geometric Methods in Theoretical Physics, 1991, New York City, Vols. 1-2, World Scientific (Singapore), 471-487 (1992). MR1225136 (95d:58149)

3. Chen, W. and Ruan, Y., A new cohomology theory of orbifold, Comm. Math. Phys., 248 (2004), no. 1, 1-31. MR2104605 (2005j:57036)

4. Connes, A.: Noncommutative differential geometry, Inst. Hautes Études Sci. Publ. Math. 62, 257-360 (1985). MR 823176 (87i:58162)

5. Dolgushev, V. and Etingof, P., Hochschild cohomology of quantized symplectic orbifolds and the Chen-Ruan cohomology, Int. Math. Res. Not. 2005, no. 27, 1657-1688. MR2152067 (2006h:53101)

6. Etingof, P., Cherednik and Hecke algebras of varieties with a finite group action, arXiv:math.QA/0406499.

7. Etingof, P. and Ginzburg, V., Symplectic reflection algebras, Calogero-Moser space, and deformed Harish-Chandra homomorphism, Invent. Math. 147 (2002), no. 2, 243-348. MR:1881922 (2003b:16021)

8. Gerstenhaber, M., On the deformation of rings and algebras. II., Ann. of Math., (2) 79 (1964) 59-103. MR0171807 (30:2034)

9. Guillemin, V. and Sternberg, S., Convexity properties of the moment mapping, Invent. Math. 67 (1982), 491-513. MR664117 (83m:58037) 
10. Hochschild, G., Kostant, B., and Rosenberg, A., Differential forms on regular affine algebras, Trans. Amer. Math. Soc., 102 (1962) 383-408. MR0142598 (26:167)

11. Halbout, G., Oudom, J., and Tang, X., Deformations of linear Poisson orbifolds, arXiv:0807.0027.

12. Kontsevich, M., Deformation quantization of Poisson manifolds, Lett. Math. Phys., 66 (2003), no. 3, 157-216. MR2062626 (2005i:53122)

13. Neumaier, N., Pflaum, M., Posthuma, H., and Tang, X., Homology of formal deformations of proper étale Lie groupoids, J. Reine Angew. Math., 593 (2006), 117-168. MR2227141 (2007c:53135)

14. Oblomkov, A., Double affine Hecke algebras of rank 1 and affine cubic surfaces, Int. Math. Res. Not., 2004, no. 18, 877-912. MR2037756 (2005j:20005)

15. Pflaum, M., Posthuma, H., Tang, X., and Tseng, H., Orbifold cup products and ring structures on Hochschild cohomologies, arXiv:0706.002\%.

16. Tang, X., Deformation Quantization of pseudo (symplectic) Poisson groupoids, Geom. Funct. Anal.16 (2006), no. 3, 731-766. . MR2238946 (2007b:53187)

17. Tang, X., Quantization of Noncommutative Poisson Manifolds, thesis, U.C. Berkeley, 2004.

18. Xu, P.: Noncommutative Poisson algebras, Amer. J. Math. 116, 101-125 (1994). MR.1262428 (94m:58246)

Institut de Mathématiques et de Mod'elisation de Montpellier I3M, UMR 5149, UniVersité de Montpellier 2, F-34095 Montpellier cedex 5, France

E-mail address: ghalbout@darboux.math.univ-montp2.fr

Department of Mathematics, Washington University, St. Louis, Missouri 63130

E-mail address: xtang@math.wustl.edu 